\title{
On a conformal quotient equation. II
}

\author{
Yuxin Ge And Guofang Wang
}

In this paper, we show that two conformal invariants $Y_{2,1}$ and $\tilde{Y}_{2,1}$ defined in (1) and (2) resp. coincide and are achieved by a conformal metric $g \in \Gamma_{2}^{+}(n>4)$, which satisfies a conformal quotient equation. The paper is a continuation of our paper [13].

\section{Introduction}

Let $\left(M, g_{0}\right)$ be a compact Riemannian manifold with metric $g_{0}$ and $\left[g_{0}\right]$ the conformal class of $g_{0}$. Let $S_{g}$ be the Schouten tensor of the metric $g$ defined by

$$
S_{g}=\frac{1}{n-2}\left(\operatorname{Ric}_{g}-\frac{R_{g}}{2(n-1)} \cdot g\right) .
$$

Here $\operatorname{Ric}_{g}$ and $R_{g}$ are the Ricci tensor and scalar curvature of a metric $g$, respectively. The importance of the Schouten tensor in conformal geometry can be viewed in the following decomposition of the Riemann curvature tensor:

$$
\operatorname{Riem}_{g}=W_{g}+S_{g} \oplus g,
$$

where (1) is the Kulkani-Nomizu product. Note that $g^{-1} \cdot W_{g}$ is the invariant in a given conformal class.

Define $\sigma_{k}(g)$ be the $\sigma_{k}$-scalar curvature or $k$-scalar curvature by

$$
\sigma_{k}(g):=\sigma_{k}\left(g^{-1} \cdot S_{g}\right),
$$

where $g^{-1} \cdot S_{g}$ is locally defined by $\left(g^{-1} \cdot S_{g}\right)_{j}^{i}=\sum_{k} g^{i k}\left(S_{g}\right)_{k j}$ and $\sigma_{k}$ is the $k$ th elementary symmetric function. Here for an $n \times n$ symmetric matrix $A$, we define $\sigma_{k}(A)=\sigma_{k}(\Lambda)$, where $\Lambda=\left(\lambda_{1}, \ldots, \lambda_{n}\right)$ is the set of eigenvalues of $A$. It is clear that $\sigma_{1}(g)$ is a constant multiple of the scalar curvature $R_{g}$. The $k$-scalar curvature $\sigma_{k}(g)$, which was first considered by Viaclovsky [33], is a natural generalization of the scalar curvature. There are many interesting works related to the $k$-scalar curvatures, see for example [5-8, 18, 19, 22-24, $27,28,34,35]$. 
Let

$$
\Gamma_{k}^{+}=\left\{\Lambda=\left(\lambda_{1}, \lambda_{2}, \ldots, \lambda_{n}\right) \in \mathbb{R}^{n} \mid \sigma_{j}(\Lambda)>0, \forall j \leq k\right\}
$$

be Garding's cone. A metric $g$ is said to be $k$-positive or simply $g \in \Gamma_{k}^{+}$if $g^{-1} \cdot S_{g} \in \Gamma_{k}^{+}$for every point $x \in M$. We call $u$ is $k$-admissible if $e^{-2 u} g_{0} \in$ $\Gamma_{k}^{+}$. Set $\mathcal{C}_{k}\left(\left[g_{0}\right]\right)=\Gamma_{k}^{+} \cap\left[g_{0}\right]$.

As in [11], we define a Yamabe-type constant

(1.1) $Y_{2,1}\left(\left[g_{0}\right]\right):= \begin{cases}\inf _{g \in \mathcal{C}_{1}\left(\left[g_{0}\right]\right)} \frac{\int \sigma_{2}(g) d \operatorname{vol}(g)}{\left(\int \sigma_{1}(g) d \operatorname{vol}(g)\right)^{\frac{n-4}{n-2}},} & \text { if } n>4, \\ \int \sigma_{2}(g) d \operatorname{vol}(g), & \text { if } n=4, \\ \sup _{g \in \mathcal{C}_{1}\left(\left[g_{0}\right]\right)} \int \sigma_{2}(g) d \operatorname{vol}(g) \times \int \sigma_{1}(g) d \operatorname{vol}(g), & \text { if } n=3 .\end{cases}$

In [11], we prove the following proposition.

Proposition 1. Let $\left(M^{n}, g_{0}\right)$ be a compact Riemannian manifold with $g_{0} \in$ $\Gamma_{1}^{+}$and $n \geq 3$. Then the conformal invariant $Y_{2,1}\left(\left[g_{0}\right]\right)$ is positive if and only if there is a conformal metric $g \in\left[g_{0}\right] \cap \Gamma_{2}^{+}$.

As in [21], we also define another Yamabe invariant in the other cone $\mathcal{C}_{2}\left(\left[g_{0}\right]\right)$ when it is not empty, that is,

$(1.2) \quad \tilde{Y}_{2,1}\left(\left[g_{0}\right]\right):= \begin{cases}\inf _{g \in \mathcal{C}_{2}\left(\left[g_{0}\right]\right)} \frac{\int \sigma_{2}(g) d \operatorname{vol}(g)}{\left(\int \sigma_{1}(g) d \operatorname{vol}(g)\right)^{\frac{n-4}{n-2}},} & \text { if } n>4, \\ \int \sigma_{2}(g) d \operatorname{vol}(g), & \text { if } n=4, \\ \sup _{g \in \mathcal{C}_{2}\left(\left[g_{0}\right]\right)} \int \sigma_{2}(g) d \operatorname{vol}(g) \times \int \sigma_{1}(g) d \operatorname{vol}(g), & \text { if } n=3 .\end{cases}$

By the definition, when the dimension $n=4$, we have $Y_{2,1}\left(\left[g_{0}\right]\right)=\tilde{Y}_{2,1}\left(\left[g_{0}\right]\right)$. In this paper, we consider $n \neq 4$. Since $\mathcal{C}_{2}\left(\left[g_{0}\right]\right) \subset \mathcal{C}_{1}\left(\left[g_{0}\right]\right)$, it is clear that $Y_{2,1}\left(\left[g_{0}\right]\right) \leq \tilde{Y}_{2,1}\left(\left[g_{0}\right]\right)$ when $n>4$ and $Y_{2,1}\left(\left[g_{0}\right]\right) \geq \tilde{Y}_{2,1}\left(\left[g_{0}\right]\right)$ when $n=3$. Hence, a natural question is to know if these two invariants are same. Here, we will give an affirmative answer under the suitable assumptions. One of our main results in this paper is 
Theorem 1. Let $\left(M^{n}, g_{0}\right)$ be a compact Riemannian manifold with $g_{0} \in \Gamma_{2}^{+}$ and $n \geq 3$. Assume that $0<Y_{2,1}\left(\left[g_{0}\right]\right)<+\infty$. Then we have

$$
Y_{2,1}\left(\left[g_{0}\right]\right)=\tilde{Y}_{2,1}\left(\left[g_{0}\right]\right)
$$

Moreover, if $n>4$, then $Y_{2,1}\left(\left[g_{0}\right]\right)$ can be achieved by some conformal metric $g \in \Gamma_{2}^{+} \cap\left[g_{0}\right]$.

In the case $n \geq 4$, the invariant $Y_{2,1}\left(\left[g_{0}\right]\right)$ is finite real number. Moreover, in the case $n \geq 5$, we have always $\tilde{Y}_{2,1}\left(M,\left[g_{0}\right]\right) \leq \tilde{Y}_{2,1}\left(\mathbb{S}^{n}\right)$, where $\tilde{Y}_{2,1}\left(\mathbb{S}^{n}\right)$ is defined for the conformal class of the standard sphere. The equality holds if and only if $M=\mathbb{S}^{n}$ is the standard sphere. Hence, the assumption $Y_{2,1}\left(\left[g_{0}\right]\right)<+\infty$ is need just for the case $n=3$. Till now we do not know if it is bounded, although we believe it is true. This is a Sobolev-type inequality. Recently, we obtained in [15] another (optimal) Sobolev-type inequality for three-dimensional (3D) manifolds. This is related to a geometric inequality, which was recently obtained by (3D) Andrews [9] and De Lellis and Topping [10]. See also $[14,16]$.

Following the definition of the sigma invariant of Schoen [30] (see also $[26])$, one can define a differential invariant by using $Y_{2,1}(n>4)$

$$
\tau_{2}(M)=\sup _{\mathcal{C}_{1}([g]) \neq \emptyset} Y_{2,1}([g])
$$

Previously, we wanted to use $\tilde{Y}_{2,1}$ to define it. The advantage to use $Y_{2,1}$ is that it might be easier to study. With Theorem 1 we know that both are the same, provided $\tau_{2}(M)>0$. One can show that

$$
\tau_{2}(M) \leq \tau_{2}\left(\mathbb{S}^{n}\right)=\tau_{2}\left(\mathbb{S}^{n-1} \times \mathbb{S}^{1}\right)
$$

We hope to use it to study the classification of 5D manifolds, as Bray and Neves [3] and Akutagawa and Neves [2] did for 3D manifolds by using the sigma invariant.

\section{Yamabe-type flows}

Set

$$
\mathcal{F}_{2}(g)=\int_{M} \sigma_{2}(g) d \operatorname{vol}(g)
$$


For any small $\varepsilon \in(0,1)$, consider the following perturbed functional:

$$
\mathcal{F}_{1, \varepsilon}(g)=\int_{M} \mathrm{e}^{2 \varepsilon u} \sigma_{1}(g) d \operatorname{vol}(g)
$$

for $g=\mathrm{e}^{-2 u} g_{0}$. The variation of $\mathcal{F}_{1, \varepsilon}$ is given

$$
\begin{aligned}
\frac{d}{d t} \mathcal{F}_{1, \varepsilon}(g)= & \frac{n-2-2 \varepsilon}{2} \int \mathrm{e}^{2 \varepsilon u} \sigma_{1}(g) g^{-1} \cdot \frac{d}{d t} g d \operatorname{vol}(g) \\
& -\frac{1}{2} \int \mathrm{e}^{2 \varepsilon u} \Delta_{g}\left(g^{-1} \cdot \frac{d}{d t} g\right) d \operatorname{vol}(g) \\
= & \frac{n-2-2 \varepsilon}{2} \int \mathrm{e}^{2 \varepsilon u} \sigma_{1}(g) g^{-1} \cdot \frac{d}{d t} g d \operatorname{vol}(g) \\
& -\frac{1}{2} \int \Delta_{g}\left(e^{2 \varepsilon u}\right) g^{-1} \cdot \frac{d}{d t} g d \operatorname{vol}(g),
\end{aligned}
$$

where $\Delta_{g}$ is the Laplacian operator with respect to $g=\mathrm{e}^{-2 u} g_{0}$. It is easy to see that

$$
\Delta_{g}\left(\mathrm{e}^{2 \varepsilon u}\right)=2 \varepsilon \mathrm{e}^{2(1+\varepsilon) u}\left(\Delta u+(2 \varepsilon-(n-2))|\nabla u|^{2}\right) .
$$

Set for $g=\mathrm{e}^{-2 u} g_{0}$

$$
\begin{aligned}
\sigma_{1, \varepsilon}(g) & =\frac{n-2-2 \varepsilon}{n-2-4 \varepsilon}\left\{\sigma_{1}(g)-\frac{2 \varepsilon}{n-2-2 \varepsilon} \mathrm{e}^{2 u}\left(\Delta u+(2 \varepsilon-(n-2))|\nabla u|^{2}\right)\right\} \\
& =\sigma_{1}(g)+\varepsilon \mathrm{e}^{2 u}\left\{|\nabla u|^{2}+\frac{2}{n-2-4 \varepsilon} \sigma_{1}\left(g_{0}\right)\right\} .
\end{aligned}
$$

From the computation given above, we have

Lemma 1. We have

$$
\frac{d}{d t} \mathcal{F}_{1, \varepsilon}(g)=\frac{n-2-4 \varepsilon}{2} \int \mathrm{e}^{2 \varepsilon u} \sigma_{1, \varepsilon}(g) g^{-1} \cdot \frac{d}{d t}(g) d \operatorname{vol}(g) .
$$

Now we introduce a flow, which non-increases (resp. non-decreases) $\mathcal{F}_{2}$ when $n \geq 4$ (resp. $n=3$ ) and preserves $\mathcal{F}_{1, \varepsilon}$.

$$
\begin{aligned}
\frac{d u}{d t}= & -\frac{1}{2} g^{-1} \frac{d}{d t} g=h_{\varepsilon}\left(\mathrm{e}^{-2 u} \frac{\sigma_{2}(g)}{\sigma_{1}(g)}\right) \\
& -h_{\varepsilon}\left(r_{\varepsilon}(g)\left(1+\frac{\mathrm{e}^{2 u} \nu}{\sigma_{1}(g)}\right) \mathrm{e}^{(2 \varepsilon-2) u}\right)+s_{\varepsilon}(g),
\end{aligned}
$$


where $r_{\varepsilon}(g)$ and $s_{\varepsilon}(g)$ are space constants, given by

$$
r_{\varepsilon}(g)=\frac{\int_{M} \sigma_{2}(g) d \operatorname{vol}(g)}{\int_{M} \mathrm{e}^{2 \varepsilon u} \sigma_{1, \varepsilon}(g) d \operatorname{vol}(g)}=\frac{n-2-4 \varepsilon}{n-2-2 \varepsilon} \frac{\int_{M} \sigma_{2}(g) d \operatorname{vol}(g)}{\int_{M} \mathrm{e}^{2 \varepsilon u} \sigma_{1}(g) d \operatorname{vol}(g)}
$$

and

$$
\begin{gathered}
\int_{M} \mathrm{e}^{2 \varepsilon u} \sigma_{1, \varepsilon}(g)\left\{h_{\varepsilon}\left(\mathrm{e}^{-2 u} \frac{\sigma_{2}(g)}{\sigma_{1}(g)}\right)-h_{\varepsilon}\left(r_{\varepsilon}(g)\left(1+\frac{\mathrm{e}^{2 u} \nu}{\sigma_{1}(g)}\right) \mathrm{e}^{(2 \varepsilon-2) u}\right)\right. \\
\left.+s_{\varepsilon}(g)\right\} d \operatorname{vol}(g)=0
\end{gathered}
$$

and

$$
\nu=\varepsilon\left(|\nabla u|^{2}+\frac{2}{n-2-4 \varepsilon} \sigma_{1}\left(g_{0}\right)\right)
$$

Here, $h_{\varepsilon}: \mathbb{R}_{+} \rightarrow \mathbb{R}$ is smooth concave function satisfying

$$
\begin{gathered}
h_{\varepsilon}(t)= \begin{cases}t, & \text { if } t \leq 1, \\
\alpha_{\varepsilon} t^{1-\frac{\varepsilon}{2}}+\beta_{\varepsilon}, & \text { if } t \geq 2,\end{cases} \\
h_{\varepsilon}^{\prime}(t)+h_{\varepsilon}^{\prime \prime}(t) t \geq 0, \quad \forall t \geq 0,
\end{gathered}
$$

where the constants $\alpha_{\varepsilon}>0, \beta_{\varepsilon}$ are bounded as $\varepsilon \rightarrow 0$ and $\alpha_{\varepsilon} \rightarrow 1$ as $\varepsilon \rightarrow 0$. From the definition, we infer

$$
h_{\varepsilon}^{\prime}(t)=\alpha_{\varepsilon}\left(1-\frac{\varepsilon}{2}\right) t^{-\frac{\varepsilon}{2}} \text { if } t \geq 2 .
$$

Lemma 2. Assume flow (2.2) stays always in the cone $\mathcal{C}_{1}\left(\left[g_{0}\right]\right)$, namely, for all $t, g(t) \in \Gamma_{1}^{+}$. Then, flow preserves $\mathcal{F}_{1, \varepsilon}$ and non-increases (resp. nondecreases) $\mathcal{F}_{2}$ when $n>4$ (resp. $n=3$ ). Hence, $r_{\varepsilon}$ is non-increasing (resp. non-decreasing) along the flow when $n>4$ (resp. $n=3$ ). 
Proof. By the definition of $s_{\varepsilon}(g)$ and Lemma 1, flow (2.2) preserves $\mathcal{F}_{1, \varepsilon}$. By the definition of $s_{\varepsilon}$ and $r_{\varepsilon}$, we can compute as follows:

$$
\begin{aligned}
- & \frac{2}{n-4} \frac{d}{d t} \mathcal{F}_{2}(g) \\
= & -\int_{M} \sigma_{2}(g) g^{-1} \cdot \frac{d}{d t} g d \operatorname{vol}(g) \\
= & -\int\left(\sigma_{2}(g)-r_{\varepsilon}(g) e^{2 \varepsilon u} \sigma_{1, \varepsilon}(g)\right) g^{-1} \cdot \frac{d}{d t} g d \operatorname{vol}(g) \\
= & 2 \int\left(\sigma_{2}(g)-r_{\varepsilon}(g) e^{2 \varepsilon u} \sigma_{1, \varepsilon}(g)\right) \\
& \times\left(h_{\varepsilon}\left(\mathrm{e}^{-2 u} \frac{\sigma_{2}(g)}{\sigma_{1}(g)}\right)-h_{\varepsilon}\left(r_{\varepsilon}(g)\left(1+\frac{\mathrm{e}^{2 u} \nu}{\sigma_{1}(g)}\right) \mathrm{e}^{(2 \varepsilon-2) u}\right)\right) d \operatorname{vol}(g) \\
= & 2 \int \mathrm{e}^{2 u} \sigma_{1}(g)\left(\mathrm{e}^{-2 u} \frac{\sigma_{2}(g)}{\sigma_{1}(g)}-r_{\varepsilon}(g)\left(1+\frac{\mathrm{e}^{2 u} \nu}{\sigma_{1}(g)}\right) \mathrm{e}^{(2 \varepsilon-2) u}\right) \\
& \times\left(h_{\varepsilon}\left(\mathrm{e}^{-2 u} \frac{\sigma_{2}(g)}{\sigma_{1}(g)}\right)-h_{\varepsilon}\left(r_{\varepsilon}(g)\left(1+\frac{\mathrm{e}^{2 u} \nu}{\sigma_{1}(g)}\right) \mathrm{e}^{(2 \varepsilon-2) u}\right)\right) d \operatorname{vol}(g) .
\end{aligned}
$$

Lemma 3 (see [11]). For $1<k \leq n$ set $F=\frac{\sigma_{k}}{\sigma_{k-1}}$. We have

(1) the matrix $\left(F^{i j}\right)(W)$ is semi-positive definite for $W \in \Gamma_{k-1}^{+}$and is positive-definite for $W \in \Gamma_{k-1}^{+} \backslash \mathcal{R}_{1}$, where $\mathcal{R}_{1}$ is the set of symmetric matrices of rank 1.

(2) The function $F$ is concave in the cone $\Gamma_{k-1}^{+}$. When $k=2$, for all $W \in$ $\Gamma_{1}^{+}$and for all $R=\left(r_{i j}\right) \in \mathcal{S}_{n}$, we have

$$
\sum_{i j k l} \frac{\partial^{2}}{\partial w_{i j} \partial w_{k l}}\left(\frac{\sigma_{2}(W)}{\sigma_{1}(W)}\right) r_{i j} r_{k l}=-\frac{\sum_{i j}\left(\sigma_{1}(W) r_{i j}-\sigma_{1}(R) w_{i j}\right)^{2}}{\sigma_{1}^{3}(W)}
$$

Set

$$
\tilde{\mathcal{F}}_{2, \varepsilon}(g)=\left(\mathcal{F}_{1, \varepsilon}\right)^{-\frac{n-4}{n-2 \varepsilon-2}} \int_{M} \sigma_{2}(g) d \operatorname{vol}(g)
$$

and

$$
\begin{aligned}
& Y_{\varepsilon}\left(M,\left[g_{0}\right]\right)=\inf _{g \in \mathcal{C}_{1}\left(\left[g_{0}\right]\right)} \tilde{\mathcal{F}}_{2, \varepsilon}(g), \\
& \tilde{Y}_{\varepsilon}\left(M,\left[g_{0}\right]\right)=\inf _{g \in \mathcal{C}_{2}\left(\left[g_{0}\right]\right)} \tilde{\mathcal{F}}_{2, \varepsilon}(g) .
\end{aligned}
$$


In the case $n>4$ and under the assumptions as in Theorem 1, the above discussion shows that (2.2) decreases the functional $\tilde{\mathcal{F}}_{2, \varepsilon}(g)$. If $g$ is a stationary point of the flow, then the metric $g$ satisfies the following perturbed equation:

$$
\frac{\sigma_{2}(g)}{\sigma_{1}(g)}-c \mathrm{e}^{2 \varepsilon u} \frac{e^{2 u} \nu}{\sigma_{1}(g)}=c \mathrm{e}^{2 \varepsilon u}
$$

or equivalently

$$
\frac{\sigma_{2}(g)}{\sigma_{1, \varepsilon}(g)}=c \mathrm{e}^{2 \varepsilon u},
$$

where $c>0$ is some positive constant. This is the perturbed equation that we use to approximate the following equation:

$$
\frac{\sigma_{2}(g)}{\sigma_{1}(g)}=1 .
$$

We will show that $Y_{\varepsilon}$ is achieved at $u_{\varepsilon} \in \mathcal{C}_{2}\left(\left[g_{0}\right]\right)$ for any small $\varepsilon>0$, which is clearly a solution of (2.12). Hence, we can conclude

$$
Y_{2,1}\left(\left[g_{0}\right]\right)=\tilde{Y}_{2,1}\left(\left[g_{0}\right]\right),
$$

since $\mathrm{e}^{-2 u_{\varepsilon}} g_{0}$ converges to the extremal metric when $\varepsilon \rightarrow 0$. Similarly, we have the same result in the case $n=3$.

\section{Local estimates}

In this section, we will study local estimates for flow (2.2) and Equation (2.12). In this paper, $C$ and $C^{\prime}$ denote positive constants, which in general are independent of $\varepsilon$. They vary from line to line. Recall $\nu=\varepsilon\left(|\nabla u|^{2}+\frac{2 \sigma_{1}\left(g_{0}\right)}{n-2-4 \varepsilon}\right)$. Note that $\nu \geq 0$ and $\sigma_{1, \varepsilon}(g)=\sigma_{1}(g)+e^{2 u} \nu$. By the standard implicit function theorem, we have the following short-time existence result. Let $T^{*} \in(0, \infty]$ be the maximum of the existence of the flow.

Theorem 2. Assume $n \geq 3$. Let $u$ be a solution of (2.2) in a geodesic ball $B_{r} \times[0, T]$ for $T<T^{*}$ and $r<r_{0}$, the injectivity radius of $M$. We suppose that the function $r_{\varepsilon}(g(t))$ is positive and bounded on $\left[0, T^{*}\right)$ and for all $t \in\left[0, T^{*}\right)$ we have $g(t)=\mathrm{e}^{-2 u(t)} g_{0} \in \Gamma_{1}^{+}$. Then there are constant $\varepsilon_{0}>0$ depending only on $\left(B_{r}, g_{0}\right)$, and constant $C$ depending only on $\left(B_{r}, g_{0}\right)$ (independent of $\varepsilon$ ) and the upper bound of $r_{\varepsilon}(g(t))$ on $[0, T]$ such that for any 
$\varepsilon \in\left(0, \varepsilon_{0}\right)$ and $(x, t) \in B_{r / 2} \times[0, T]$

$$
|\nabla u|^{2}+\left|\nabla^{2} u\right| \leq C\left(1+e^{-\frac{2-2 \varepsilon}{1-\varepsilon / 2} \inf _{(x, t) \in B_{r} \times[0, T]} u(x, t)}\right) .
$$

Proof. Let $W=\left(w_{i j}\right)$ be an $n \times n$ matrix with $w_{i j}=\nabla_{i j}^{2} u+u_{i} u_{j}-\frac{|\nabla u|^{2}}{2}$ $\left(g_{0}\right)_{i j}+\left(S_{g_{0}}\right)_{i j}$. Here $u_{i}$ and $u_{i j}$ are the first and second derivatives of $u$ with respect to the background metric $g_{0}$. Set $K=r_{\varepsilon}(g(t)) \mathrm{e}^{(2 \varepsilon-2) u}$ and $K_{1}=K\left(1+\frac{\nu}{\sigma_{1}(W)}\right)$. Let $F_{\varepsilon}: \Gamma_{+}^{1} \times \mathbb{R}^{+} \times \mathbb{R} \times \mathbb{R}^{+} \rightarrow \mathbb{R}$ be regular function defined by

$$
\begin{aligned}
F_{\varepsilon}(W, \nu, u, t) & :=h_{\varepsilon}\left(\frac{\sigma_{2}(W)}{\sigma_{1}(W)}\right)-h_{\varepsilon}\left(r_{\varepsilon}(g(t))\left(1+\frac{\nu}{\sigma_{1}(W)}\right) e^{(2 \varepsilon-2) u}\right) \\
& =h_{\varepsilon}\left(\frac{\sigma_{2}(W)}{\sigma_{1}(W)}\right)-h_{\varepsilon}\left(K_{1}\right) .
\end{aligned}
$$

Set

$$
\begin{aligned}
F_{\varepsilon}^{i j}(W, \nu, u, t) & :=\frac{\partial F_{\varepsilon}}{\partial w_{i j}}(W, \nu, u, t) \\
& =h_{\varepsilon}^{\prime}\left(\frac{\sigma_{2}(W)}{\sigma_{1}(W)}\right) \frac{\sigma_{1}(W) T^{i j}-\sigma_{2}(W) \delta^{i j}}{\sigma_{1}^{2}(W)}+h_{\varepsilon}^{\prime}\left(K_{1}\right) \frac{K \nu \delta^{i j}}{\sigma_{1}^{2}(W)},
\end{aligned}
$$

where $\left(T^{i j}\right)=\left(\sigma_{1}(W) \delta^{i j}-w^{i j}\right)$ is the first Newton transformation associated with $W$, and $\delta^{i j}$ is the Kronecker symbol. As $W \in \Gamma_{1}^{+}$and $h_{\varepsilon}^{\prime}$ is positive on $(0,+\infty)$, we see that $\left(F_{\varepsilon}^{i j}\right)$ is positive definite.

Lemma 4. $F_{\varepsilon}$ is concave in $\Gamma_{1}^{+}$.

Proof. To show this, we compute

$$
\begin{aligned}
\frac{\partial^{2} F_{\varepsilon}}{\partial w_{i j} w_{k l}}= & h_{\varepsilon}^{\prime \prime}\left(\frac{\sigma_{2}(W)}{\sigma_{1}(W)}\right) \frac{\partial}{\partial w_{i j}}\left(\frac{\sigma_{2}(W)}{\sigma_{1}(W)}\right) \frac{\partial}{\partial w_{k l}}\left(\frac{\sigma_{2}(W)}{\sigma_{1}(W)}\right) \\
& +h_{\varepsilon}^{\prime}\left(\frac{\sigma_{2}(W)}{\sigma_{1}(W)}\right) \frac{\partial^{2}}{\partial w_{i j} \partial w_{k l}}\left(\frac{\sigma_{2}(W)}{\sigma_{1}(W)}\right) \\
& -h_{\varepsilon}^{\prime \prime}\left(K_{1}\right)\left(\frac{K \nu}{\sigma_{1}^{2}(W)}\right)^{2} \delta^{i j} \delta^{k l}-2 h_{\varepsilon}^{\prime}\left(K_{1}\right) \frac{K \nu}{\sigma_{1}^{3}(W)} \delta^{i j} \delta^{k l} \\
:= & \mathrm{I}+\mathrm{II}
\end{aligned}
$$


where

$$
\begin{aligned}
I:= & h_{\varepsilon}^{\prime \prime}\left(\frac{\sigma_{2}(W)}{\sigma_{1}(W)}\right) \frac{\partial}{\partial w_{i j}}\left(\frac{\sigma_{2}(W)}{\sigma_{1}(W)}\right) \frac{\partial}{\partial w_{k l}}\left(\frac{\sigma_{2}(W)}{\sigma_{1}(W)}\right) \\
& +h_{\varepsilon}^{\prime}\left(\frac{\sigma_{2}(W)}{\sigma_{1}(W)}\right) \frac{\partial^{2}}{\partial w_{i j} \partial w_{k l}}\left(\frac{\sigma_{2}(W)}{\sigma_{1}(W)}\right)
\end{aligned}
$$

and

$$
\mathrm{II}:=-h_{\varepsilon}^{\prime \prime}\left(K_{1}\right)\left(\frac{K \nu}{\sigma_{1}^{2}(W)}\right)^{2} \delta^{i j} \delta^{k l}-2 h_{\varepsilon}^{\prime}\left(K_{1}\right) \frac{K \nu}{\sigma_{1}^{3}(W)} \delta^{i j} \delta^{k l} .
$$

Recall that $h_{\varepsilon}$ is concave and by lemma $3, \frac{\sigma_{2}(W)}{\sigma_{1}(W)}$ is concave in $W$ in the cone $\Gamma_{+}^{1}$. Thus $(I)$, as a matrix, is non-positive definite. On the other hand, it follows from $(2.7)$ we have

$$
\left(\left[-h_{\varepsilon}^{\prime \prime}\left(K_{1}\right) \frac{K \nu}{\sigma_{1}(W)}-h_{\varepsilon}^{\prime}\left(K_{1}\right)\right] \frac{K \nu}{\sigma_{1}^{3}(W)} \delta^{i j} \delta^{k l}\right) \leq 0,
$$

since $K>0, \nu>0, h_{\varepsilon}^{\prime}(t)+h_{\varepsilon}^{\prime \prime}(t) t \geq 0$ for all $t \geq 0$ and $\sigma_{1}(W)>0$. Therefore, we prove the lemma.

From the proof of Lemma 4, we in fact have

$$
\sum_{i j k l} \frac{\partial^{2} F_{\varepsilon}}{\partial w_{i j} \partial w_{k l}} r^{i j} r^{k l} \leq-h_{\varepsilon}^{\prime}\left(K_{1}\right) \frac{K \nu}{\sigma_{1}^{3}(W)}\left(\sum_{i} r^{i i}\right)^{2}
$$

For the simplicity of notations, we now drop the index $\varepsilon$, if there is no confusion. We try to show the local estimates for first- and second-order derivatives together. Let $S(T M)$ denote the unit tangent bundle of $M$ with respect to the background metric $g_{0}$. We define a function $\tilde{G}: S(T M) \times$ $[0, T] \rightarrow \mathbb{R}$

$$
\tilde{G}(e, t)=\left(\nabla^{2} u+|\nabla u|^{2} g_{0}\right)(e, e) \text {. }
$$

Without loss of generality, we assume $r=1$. Let $\rho \in C_{0}^{\infty}\left(B_{1}\right)$ be a cut-off function defined as in [19] such that

$$
\begin{aligned}
\rho & \geq 0, & & \text { in } B_{1}, \\
\rho & =1, & & \text { in } B_{1 / 2}, \\
|\nabla \rho(x)| & \leq 2 b_{0} \rho^{1 / 2}(x), & & \text { in } B_{1}, \\
\left|\nabla^{2} \rho\right| & \leq b_{0}, & & \text { in } B_{1} .
\end{aligned}
$$


Here, $b_{0}>1$ is a constant. Since $\mathrm{e}^{-2 u} g_{0} \in \Gamma_{1}^{+}$, to bound $|\nabla u|$ and $\left|\nabla^{2} u\right|$, we only need to bound $\left(\nabla^{2} u+|\nabla u|^{2} g_{0}\right)(e, e)$ from above for all $e \in S(T M)$ and for all $t \in[0, T]$. To see this, denote $G(e, t)=\rho(x) \tilde{G}(e, t)$. Assume $x_{0} \in M$ and $\left(e_{1}, t_{0}\right) \in S\left(T_{x_{0}} M\right) \times[0, T]$ such that

$$
\begin{gathered}
G\left(e_{1}, t_{0}\right)=\max _{S(T M) \times[0, T]} G(e, t), \\
t_{0}>0 \\
G\left(e_{1}, t_{0}\right)>n \max _{B_{1}} \sigma_{1}\left(g_{0}\right) .
\end{gathered}
$$

Let $\left(e_{1}, \ldots, e_{n}\right)$ be a orthonormal basis at point $\left(x_{0}, t_{0}\right)$. It follows from the fact $W \in \Gamma_{1}^{+}$

$$
\begin{aligned}
n G\left(e_{1}, t_{0}\right) & \geq \rho\left(\Delta u+n|\nabla u|^{2}\right) \geq \rho\left(n|\nabla u|^{2}+\frac{n-2}{2}|\nabla u|^{2}-\sigma_{1}\left(g_{0}\right)\right) \\
& \geq \frac{3 n-2}{2} \rho|\nabla u|^{2}-\frac{1}{n} G\left(e_{1}, t_{0}\right),
\end{aligned}
$$

so that

$$
G\left(e_{1}, t_{0}\right) \geq \frac{\frac{3 n-2}{2}}{n+\frac{1}{n}} \rho|\nabla u|^{2} \geq \frac{21}{20} \rho|\nabla u|^{2} .
$$

Consequently, we obtain

$$
\nabla_{11}^{2} u\left(x_{0}, t_{0}\right) \geq \frac{1}{20}|\nabla u|^{2}\left(x_{0}, t_{0}\right) .
$$

Set for any $i \neq j \in\{1, \ldots, n\}$

$$
e^{\prime}=\frac{1}{\sqrt{2}}\left(e_{i} \pm e_{j}\right)
$$

We have

$$
G\left(e^{\prime}, t_{0}\right)=\frac{1}{2}\left(G\left(e_{i}, t_{0}\right)+G\left(e_{j}, t_{0}\right)\right) \pm \rho \nabla_{i j}^{2} u\left(x_{0}, t_{0}\right) .
$$

Thus, there holds

$$
\rho\left|\nabla_{i j}^{2} u\left(x_{0}, t_{0}\right)\right| \leq G\left(e_{1}, t_{0}\right)-\frac{1}{2}\left(G\left(e_{i}, t_{0}\right)+G\left(e_{j}, t_{0}\right)\right) .
$$


On the other hand, we have $\forall i=1, \ldots, n$

$$
\begin{aligned}
(n-1) G\left(e_{1}, t_{0}\right)+G\left(e_{i}, t_{0}\right) & \geq \rho\left(\Delta u+n|\nabla u|^{2}\right) \\
& \geq \rho\left(\frac{3 n-2}{2}|\nabla u|^{2}-\sigma_{1}\left(g_{0}\right)\right),
\end{aligned}
$$

which implies

$$
G\left(e_{i}, t_{0}\right) \geq \rho\left(\frac{3 n-2}{2}|\nabla u|^{2}-\sigma_{1}\left(g_{0}\right)\right)-(n-1) G\left(e_{1}, t_{0}\right) .
$$

Together with (3.12), we deduce

$$
\rho\left|\nabla_{i j}^{2} u\left(x_{0}, t_{0}\right)\right| \leq n G\left(e_{1}, t_{0}\right)-\frac{3 n-2}{2} \rho|\nabla u|^{2}+\rho \sigma_{1}\left(g_{0}\right) \leq(n+1) G\left(e_{1}, t_{0}\right) .
$$

(indeed, at all point $(x, t)$, the estimate $\rho\left|\nabla_{i j}^{2} u\right| \leq(n+1) G\left(e_{1}, t_{0}\right)$ holds). Now choose the normal coordinates around $x_{0}$ such that at point $x_{0}$

$$
\frac{\partial}{\partial x_{1}}=e_{1}
$$

and consider the function on $M \times[0, T]$

$$
G(x, t)=\rho(x)\left(u_{11}+|\nabla u|^{2}\right)(x, t)
$$

(without the confusion, we denotes also this function by $G$ ). Clearly, $\left(x_{0}, t_{0}\right)$ is a maximum point of $G(x, t)$ on $M \times[0, T]$. At $\left(x_{0}, t_{0}\right)$, we have

(3.16) $0 \leq G_{t}=\rho\left(u_{11 t}+2 \sum_{l} u_{l} u_{l t}\right)$,

(3.17) $0=G_{j}=\frac{\rho_{j}}{\rho} G+\rho\left(u_{11 j}+2 \sum_{l \geq 1} u_{l} u_{l j}\right), \quad$ for any $j$,

(3.18) $0 \geq\left(G_{i j}\right)=\left(\frac{\rho \rho_{i j}-2 \rho_{i} \rho_{j}}{\rho^{2}} G+\rho\left(u_{11 i j}+\sum_{l \geq 1}\left(2 u_{l i} u_{l j}+2 u_{l} u_{l i j}\right)\right)\right)$. 
Recall that $\left(F^{i j}\right)$ is definite positive. Hence, we have

$$
\begin{aligned}
0 \geq & \sum_{i, j \geq 1} F^{i j} G_{i j}-G_{t} \\
\geq & \sum_{i, j \geq 1} F^{i j} \frac{\rho \rho_{i j}-2 \rho_{i} \rho_{j}}{\rho^{2}} G+\rho \sum_{i, j \geq 1} F^{i j}\left(u_{11 i j}+\sum_{l \geq 1}\left(2 u_{l i} u_{l j}+2 u_{l} u_{l i j}\right)\right) \\
& -\rho\left(u_{11 t}+2 \sum_{l \geq 1} u_{l} u_{l t}\right)
\end{aligned}
$$

First, from the definition of $\rho$, we have

$$
\sum_{i, j \geq 1} F^{i j} \frac{\rho \rho_{i j}-2 \rho_{i} \rho_{j}}{\rho^{2}} G \geq-C \sum_{i, j \geq 1}\left|F^{i j}\right| \frac{1}{\rho} G
$$

and

$$
\sum_{i, j \geq 1}\left|F^{i j}\right| \geq \sum_{i} F^{i i} \geq C \sum_{i, j \geq 1}\left|F^{i j}\right|
$$

since $F$ is positive definite. Using the facts that

$$
\begin{aligned}
u_{k i j}= & u_{i j k}+\sum_{m} R_{m i k j} u_{m} \\
u_{k k i j}= & u_{i j k k}+\sum_{m}\left(2 R_{m i k j} u_{m k}-R i c_{m j} u_{m i}\right. \\
& \left.-R i c_{m i} u_{m j}-R i c_{m i, j} u_{m}+R_{m i k j, k} u_{m}\right)
\end{aligned}
$$

and

$$
\left(\sum_{l} u_{l}^{2}\right)_{11}=2 \sum_{l}\left(u_{11 l} u_{l}+u_{1 l}^{2}\right)+O\left(|\nabla u|^{2}\right)
$$


we have

$$
\begin{aligned}
& \sum_{i, j \geq 1} F^{i j} u_{11 i j} \\
& \quad \geq \sum_{i, j \geq 1} F^{i j}\left(w_{i j 11}-\left(u_{11}\right)_{i} u_{j}-u_{i}\left(u_{11}\right)_{j}+\sum_{l \geq 1}\left(u_{1 l}^{2}+u_{11 l} u_{l}\right)\left(g_{0}\right)_{i j}\right) \\
& \quad-2 \sum_{i, j \geq 1} F^{i j} u_{i 1} u_{j 1}-C\left(1+\left|\nabla^{2} u\right|+|\nabla u|^{2}\right) \sum_{i, j \geq 1}\left|F^{i j}\right|
\end{aligned}
$$

and

$$
\begin{aligned}
\sum_{i, j, l} F^{i j} u_{l} u_{l i j} \geq & \sum_{i, j, l} F^{i j} u_{l} w_{i j l}-\sum_{i, j, l} F^{i j}\left(u_{l} u_{i l} u_{j}+u_{l} u_{i} u_{j l}\right) \\
& +\frac{1}{2} \sum_{i, j} F^{i j}\left\langle\nabla u, \nabla\left(|\nabla u|^{2}\right)\right\rangle\left(g_{0}\right)_{i j}-C\left(1+|\nabla u|^{2}\right) \sum_{i, j \geq 1}\left|F^{i j}\right|
\end{aligned}
$$

Combining (3.25) and (3.26), we deduce

$$
\begin{aligned}
\sum_{i, j \geq 1} & F^{i j}\left(u_{11 i j}+2 \sum_{l \geq 1}\left(u_{l i} u_{l j}+u_{l} u_{l i j}\right)\right) \\
\geq & \sum_{i, j \geq 1} F^{i j}\left(w_{i j 11}+2 \sum_{l \geq 1} w_{i j l} u_{l}\right)+2 \sum_{i, j \geq 1} F^{i j} \sum_{l \geq 2} u_{l i} u_{l j}+\sum_{i, j, l \geq 1} u_{1 l}^{2} F^{i j}\left(g_{0}\right)_{i j} \\
& -\sum_{i, j} F^{i j}\left[\left(u_{11}+|\nabla u|^{2}\right)_{i} u_{j}+u_{i}\left(u_{11}+|\nabla u|^{2}\right)_{j}\right. \\
& \left.-\left\langle\nabla u, \nabla\left(u_{11}+|\nabla u|^{2}\right)\right\rangle\left(g_{0}\right)_{i j}\right] \\
& -C\left(1+\left|\nabla^{2} u\right|+|\nabla u|^{2}\right) \sum_{i, j \geq 1}\left|F^{i j}\right| \\
\geq & \sum_{i, j} F^{i j}\left(w_{i j 11}+2 \sum_{l} w_{i j l} u_{l}\right)+u_{11}^{2} \sum_{i, j} F^{i j}\left(g_{0}\right)_{i j} \\
& +\sum_{i, j} F^{i j}\left(\rho_{i} u_{j}+\rho_{j} u_{i}-\langle\nabla \rho, \nabla u\rangle\left(g_{0}\right)_{i j} \frac{G}{\rho^{2}}\right. \\
& -C\left(1+\left|\nabla^{2} u\right|+|\nabla u|^{2}\right) \sum_{i, j \geq 1}\left|F^{i j}\right| .
\end{aligned}
$$


Now, we want to estimate $\sum_{i, j, l} F^{i j} w_{i j l} u_{l}$ and $\sum_{i, j} F^{i j} w_{i j 11}$, respectively. For the first term $\sum_{i, j, l} F^{i j} w_{i j l} u_{l}$, we have

$$
\begin{aligned}
\sum_{i, j, l} F^{i j} w_{i j l} u_{l}= & \sum_{l} F_{l} u_{l}+h^{\prime}\left(K_{1}\right) \frac{K}{\sigma_{1}(W)} \sum_{l} \nu_{l} u_{l} \\
& +h^{\prime}\left(K_{1}\right) K_{1}(2 \varepsilon-2) \sum_{l} u_{l}^{2} .
\end{aligned}
$$

For the second term $\sum_{i, j, l} F^{i j} w_{i j 11}$, we have

$$
\begin{aligned}
\sum_{i, j} F^{i j} w_{i j 11}= & F_{11}-\sum_{i, j, k, m} \frac{\partial^{2} F}{\partial w_{i j} \partial w_{k m}} w_{i j 1} w_{k m 1} \\
& -2 \sum_{i, j} \frac{\partial^{2} F}{\partial w_{i j} \partial u} w_{i j 1} u_{1}-2 \sum_{i, j} \frac{\partial^{2} F}{\partial w_{i j} \partial \nu} w_{i j 1} \nu_{1} \\
& -\frac{\partial^{2} F}{\partial \nu^{2}} \nu_{1}^{2}-2 \frac{\partial^{2} F}{\partial \nu \partial u} \nu_{1} u_{1}-\frac{\partial^{2} F}{\partial u^{2}} u_{1}^{2}-\frac{\partial F}{\partial \nu} \nu_{11}-\frac{\partial F}{\partial u} u_{11} .
\end{aligned}
$$

It follows from (3.4) that

$$
-\sum_{i, j, k, m} \frac{\partial^{2} F}{\partial w_{i j} \partial w_{k m}} w_{i j 1} w_{k m 1} \geq h^{\prime}\left(K_{1}\right) \frac{K \nu}{\sigma_{1}^{3}(W)}\left(\sum_{i} w_{i i 1}\right)^{2} .
$$

Using the facts (2.6)-(2.8), we can estimate successively

$$
\begin{aligned}
& -2 \sum_{i, j} \frac{\partial^{2} F}{\partial w_{i j} \partial u} w_{i j 1} u_{1} \\
& \geq-2(2-2 \varepsilon) h^{\prime}\left(K_{1}\right) \frac{K \nu}{\sigma_{1}^{2}(W)}\left[2 \sigma_{1}(W) u_{1}^{2}+\frac{1}{8 \sigma_{1}(W)}\left(\sum_{i} w_{i i 1}\right)^{2}\right]
\end{aligned}
$$

$$
\begin{aligned}
& -2 \sum_{i, j} \frac{\partial^{2} F}{\partial w_{i j} \partial \nu} w_{i j 1} \nu_{1} \\
& \geq-2 h^{\prime}\left(K_{1}\right) \frac{K}{\sigma_{1}^{2}(W)}\left[\frac{\sigma_{1}(W)}{\nu} \nu_{1}^{2}+\frac{\nu}{4 \sigma_{1}(W)}\left(\sum_{i} w_{i i 1}\right)^{2}\right]
\end{aligned}
$$




$$
\begin{gathered}
-2 \frac{\partial^{2} F}{\partial \nu \partial u} \nu_{1} u_{1} \geq-2 h^{\prime}\left(K_{1}\right) \frac{K \nu}{\sigma_{1}(W)} u_{1}^{2}-2 h^{\prime}\left(K_{1}\right) \frac{K}{\nu \sigma_{1}(W)} \nu_{1}^{2}, \\
-\frac{\partial^{2} F}{\partial \nu^{2}} \nu_{1}^{2}=h^{\prime \prime}\left(K_{1}\right) \frac{K^{2}}{\sigma_{1}^{2}(W)} \nu_{1}^{2} \geq-h^{\prime}\left(K_{1}\right) \frac{K}{\sigma_{1}^{2}(W)} \nu_{1}^{2} \\
-\frac{\partial^{2} F}{\partial u^{2}} u_{1}^{2} \geq 0
\end{gathered}
$$

and

$$
2 h^{\prime}\left(K_{1}\right) \frac{K}{\sigma_{1}(W)} \sum_{l} \nu_{l} u_{l} \geq-h^{\prime}\left(K_{1}\right) \frac{K}{\sigma_{1}(W)}\left[\frac{|\nabla \nu|^{2}}{\nu}+\nu|\nabla u|^{2}\right]
$$

These estimates, together with (3.28) and (3.29), imply

$$
\begin{aligned}
\sum_{i, j} & F^{i j}\left(w_{i j 11}+2 \sum_{l} w_{i j l} u_{l}\right) \\
\geq & \left(F_{11}+\sum_{l} 2 F_{l} u_{l}\right)-11 h^{\prime}\left(K_{1}\right) \frac{K \nu}{\sigma_{1}(W)}|\nabla u|^{2} \\
& -5 h^{\prime}\left(K_{1}\right) \frac{K}{\sigma_{1}(W) \nu}|\nabla \nu|^{2}-h^{\prime}\left(K_{1}\right) \frac{K}{\sigma_{1}^{2}(W)}|\nabla \nu|^{2} \\
& -4 h^{\prime}\left(K_{1}\right) K_{1}|\nabla u|^{2}+h^{\prime}\left(K_{1}\right) \frac{K}{\sigma_{1}(W)} \nu_{11}-(2-2 \varepsilon) h^{\prime}\left(K_{1}\right) K_{1} u_{11} \\
\geq & F_{11}+\sum_{l} 2 F_{l} u_{l}-15 h^{\prime}\left(K_{1}\right) \frac{K \nu}{\sigma_{1}(W)}|\nabla u|^{2} \\
& -5 h^{\prime}\left(K_{1}\right) \frac{K}{\sigma_{1}(W) \nu}|\nabla \nu|^{2}-h^{\prime}\left(K_{1}\right) \frac{K}{\sigma_{1}^{2}(W)}|\nabla \nu|^{2} \\
& -C\left(1+e^{(2 \varepsilon-2) u} \frac{G}{\rho}+h^{\prime}\left(K_{1}\right) \frac{K}{\sigma_{1}(W)} \nu_{11}-2 h^{\prime}\left(K_{1}\right) \frac{K \nu}{\sigma_{1}(W)} u_{11} .\right.
\end{aligned}
$$

Here, we the fact that $u_{11} \geq 0$ at the point $\left(x_{0}, t_{0}\right)$. Remark

$$
\begin{aligned}
\left|\sum_{l} u_{i l} u_{l}\right| & =\left|\sum_{l}\left(w_{i l}-u_{i} u_{l}+\frac{|\nabla u|^{2}}{2}\left(g_{0}\right)_{i l}-\left(S_{g_{0}}\right)_{i l}\right) u_{l}\right| \\
& \leq \sqrt{\sigma_{1}^{2}(W)-2 \sigma_{2}(W)}|\nabla u|+\frac{|\nabla u|^{3}}{2}+\left|S_{g_{0}}\right||\nabla u|,
\end{aligned}
$$


so that together with (3.17) and (3.24), there holds

$$
\begin{aligned}
& h^{\prime}\left(K_{1}\right) \frac{K}{\sigma_{1}(W)} \nu_{11} \\
& \quad=\varepsilon h^{\prime}\left(K_{1}\right) \frac{K}{\sigma_{1}(W)}\left(|\nabla u|^{2}+\frac{2}{n-2-4 \varepsilon} \sigma_{1}\left(g_{0}\right)\right)_{11} \\
& \quad=\varepsilon h^{\prime}\left(K_{1}\right) \frac{K}{\sigma_{1}(W)}\left[2 \sum_{l}\left(\frac{-G \rho_{l} u_{l}}{\rho^{2}}-2 \sum_{j} u_{j} u_{j l} u_{l}+u_{1 l}^{2}\right)+O\left(|\nabla u|^{2}+1\right)\right] \\
& \quad \geq \varepsilon h^{\prime}\left(K_{1}\right) \frac{K}{\sigma_{1}(W)}\left[\frac{-2 G\langle\nabla \rho, \nabla u\rangle}{\rho^{2}}-C|\nabla u|^{2}\left|\nabla^{2} u\right|-C\left(|\nabla u|^{4}+1\right)\right] .
\end{aligned}
$$

Finally, we deduce that

$$
\begin{aligned}
& \sum_{i, j} F^{i j}\left(w_{i j 11}+2 \sum_{l} w_{i j l} u_{l}\right) \\
& \geq F_{11}+2 \sum_{l} F_{l} u_{l}-15 h^{\prime}\left(K_{1}\right) \frac{K \nu}{\sigma_{1}(W)}|\nabla u|^{2}-5 h^{\prime}\left(K_{1}\right) \frac{K}{\sigma_{1}(W) \nu}|\nabla \nu|^{2} \\
&-2 h^{\prime}\left(K_{1}\right) \frac{K \nu}{\sigma_{1}(W)} u_{11}-h^{\prime}\left(K_{1}\right) \frac{K}{\sigma_{1}^{2}(W)}|\nabla \nu|^{2} \\
&-C\left(1+\mathrm{e}^{(2 \varepsilon-2) u}\right) \frac{G}{\rho}-C \varepsilon h^{\prime}\left(K_{1}\right) \frac{K}{\sigma_{1}(W)}\left[\frac{G|\nabla u|}{\rho^{3 / 2}}+\frac{G}{\rho}\left(1+|\nabla u|^{2}\right)\right]
\end{aligned}
$$

Now, we claim that there is a constant $C>0$ independent of $\varepsilon$ such that

$$
G \leq C\left[1+\sqrt{\rho}\left(\sum_{i} F^{i i}\right)^{-1}\left(1+\mathrm{e}^{(2 \varepsilon-2) u}\right)+\varepsilon \rho\left(1+\mathrm{e}^{(2 \varepsilon-2) u}\right)\right]
$$

We divide the proof of the claim into two cases.

Case 1. $\frac{\nu}{\sigma_{1}(W)} \geq 1$.

It is clear that

$$
C^{\prime} \varepsilon\left(1+|\nabla u|^{2}\right) \leq \nu \leq C \varepsilon\left(1+|\nabla u|^{2}\right)
$$


and

$$
|\nabla \nu|^{2} \leq C \varepsilon \nu \frac{G^{2}}{\rho^{2}}
$$

for some positive constants $C^{\prime}$ and $C$ with $C^{\prime}<C$. Recall

$$
\sum_{i} F^{i i}=h^{\prime}\left(\frac{\sigma_{2}(W)}{\sigma_{1}(W)}\right)\left(n-1-\frac{n \sigma_{2}(W)}{\sigma_{1}^{2}(W)}\right)+h^{\prime}\left(K_{1}\right) \frac{n K \nu}{\sigma_{1}^{2}(W)}
$$

Thus, we can obtain that

$$
\begin{aligned}
& h^{\prime}\left(K_{1}\right) \frac{K \nu}{\sigma_{1}(W)}|\nabla u|^{2} \leq\left(\sum_{i} F^{i i}\right) \frac{\sigma_{1}(W)}{n}|\nabla u|^{2} \leq\left(\sum_{i} F^{i i}\right) \frac{\nu}{n}|\nabla u|^{2} \\
& \leq C \varepsilon\left(\sum_{i} F^{i i}\right) \frac{G^{2}}{\rho^{2}}, \\
& h^{\prime}\left(K_{1}\right) \frac{K \nu}{\sigma_{1}(W)} u_{11} \leq C \varepsilon\left(\sum_{i} F^{i i}\right) \frac{G^{2}}{\rho^{2}}, \\
& h^{\prime}\left(K_{1}\right) \frac{K}{\nu \sigma_{1}(W)}|\nabla \nu|^{2} \leq h^{\prime}\left(K_{1}\right) \frac{K}{\sigma_{1}^{2}(W)}|\nabla \nu|^{2} \leq C \varepsilon\left(\sum_{i} F^{i i}\right) \frac{G^{2}}{\rho^{2}}
\end{aligned}
$$

and

$$
C \varepsilon h^{\prime}\left(K_{1}\right) \frac{K}{\sigma_{1}(W)}\left[\frac{G|\nabla u|}{\rho^{3 / 2}}+\frac{G\left(1+|\nabla u|^{2}\right)}{\rho}\right] \leq C \varepsilon\left(\sum_{i} F^{i i}\right)\left[\frac{G^{3 / 2}}{\rho^{2}}+\frac{G^{2}}{\rho^{2}}\right] \text {. }
$$

Combining (3.40) and (3.45)-(3.48), we obtain

$$
\begin{aligned}
& \sum_{i, j} F^{i j}\left(w_{i j 11}+2 \sum_{l} w_{i j l} u_{l}\right) \\
& \quad \geq F_{11}+2 \sum_{l} F_{l} u_{l}-C \varepsilon\left(\sum_{i} F^{i i}\right)\left(\frac{G}{\rho}\right)^{2}-C\left(1+\mathrm{e}^{(2 \varepsilon-2) u}\right) \frac{G}{\rho},
\end{aligned}
$$


so that it follows from (3.19) to (3.21) and (3.27)

$$
\begin{aligned}
0 \geq & -C\left(\sum_{i} F^{i i}\right) \frac{G}{\rho}+\rho u_{11}^{2}\left(\sum_{i} F^{i i}\right)-C\left(\sum_{i} F^{i i}\right) \frac{G \sqrt{G}}{\rho} \\
& -C\left(\sum_{i} F^{i i}\right) G-C \varepsilon\left(\sum_{i} F^{i i}\right) \frac{G^{2}}{\rho}-C\left(1+\mathrm{e}^{(2 \varepsilon-2) u}\right) G .
\end{aligned}
$$

Therefore, we prove (3.41), provided $\varepsilon$ is sufficiently small.

Case 2. $\frac{\nu}{\sigma_{1}(W)}<1$.

We distinguish two cases.

(a) $\sigma_{2}(W) \geq 0$.

Then from (3.38), we have

$$
|\nabla \nu| \leq C \varepsilon\left(\sigma_{1}(W)|\nabla u|+|\nabla u|^{3}+|\nabla u|+1\right) .
$$

In view of (3.42), we have

$$
\begin{aligned}
& h^{\prime}\left(K_{1}\right) \frac{K \nu}{\sigma_{1}(W)}|\nabla u|^{2}+h^{\prime}\left(K_{1}\right) \frac{K}{\sigma_{1}(W) \nu}|\nabla \nu|^{2}+h^{\prime}\left(K_{1}\right) \frac{K}{\sigma_{1}^{2}(W)}|\nabla \nu|^{2} \\
& \quad+h^{\prime}\left(K_{1}\right) \frac{K \nu}{\sigma_{1}(W)}\left|u_{11}\right| \leq C\left(1+e^{(2 \varepsilon-2) u}\right) \frac{G}{\rho}
\end{aligned}
$$

and

$$
C \varepsilon h^{\prime}\left(K_{1}\right) \frac{K}{\sigma_{1}(W)}\left[\frac{G|\nabla u|}{\rho^{3 / 2}}+\frac{G}{\rho}\left(1+|\nabla u|^{2}\right)\right] \leq C\left(1+\mathrm{e}^{(2 \varepsilon-2) u}\right) \frac{G}{\rho^{3 / 2}} .
$$

Hence, we also infer (3.41), provided $\varepsilon$ is sufficiently small.

(b) $\sigma_{2}(W)<0$.

We also have (3.53) and

$$
h^{\prime}\left(K_{1}\right) \frac{K \nu}{\sigma_{1}(W)}|\nabla u|^{2}+h^{\prime}\left(K_{1}\right) \frac{K \nu}{\sigma_{1}(W)}\left|u_{11}\right| \leq C\left(1+e^{(2 \varepsilon-2) u}\right) \frac{G}{\rho} .
$$

Thanks of (3.38), we have

$$
|\nabla \nu| \leq C \varepsilon\left(\sqrt{\sigma_{1}^{2}(W)-2 \sigma_{2}(W)}|\nabla u|+|\nabla u|^{3}+|\nabla u|+1\right),
$$


so that together with (3.42) and (3.44) we obtain

$$
\begin{aligned}
h^{\prime}\left(K_{1}\right) \frac{K}{\sigma_{1}^{2}(W)}|\nabla \nu|^{2} & \leq h^{\prime}\left(K_{1}\right) \frac{K}{\sigma_{1}(W) \nu}|\nabla \nu|^{2} \\
& \leq C h^{\prime}\left(K_{1}\right) K \varepsilon^{2}\left(\frac{\sigma_{1}^{2}(W)-2 \sigma_{2}(W)}{\sigma_{1}(W) \nu}|\nabla u|^{2}+\frac{|\nabla u|^{6}+1}{\nu^{2}}\right) \\
& \leq C\left(1+e^{(2 \varepsilon-2) u}\right) \frac{G}{\rho}+C \varepsilon K \sigma_{1}(W)\left(\sum_{i} F^{i i}\right) \\
& \leq C\left(1+e^{(2 \varepsilon-2) u}\right) \frac{G}{\rho}\left(1+\varepsilon \sum_{i} F^{i i}\right) .
\end{aligned}
$$

Finally, we imply that the claim (3.41) holds in this case, provided $\varepsilon$ is sufficiently small. It is easy to see from $(2.6)$ that $h_{\varepsilon}^{\prime}(2)$ is uniformly bounded from below by a positive constant for all $\varepsilon \in[0,1 / 2]$. Hence, we have

$$
\left(\sum_{i} F^{i i}\right)^{-1} \leq \begin{cases}C, & \text { if } \frac{\sigma_{2}(W)}{\sigma_{1}(W)} \leq 2 \\ C\left(\frac{G}{\rho}\right)^{\varepsilon / 2}, & \text { if } \frac{\sigma_{2}(W)}{\sigma_{1}(W)} \geq 2\end{cases}
$$

which, together with (3.41), implies that

$$
G \leq \begin{cases}C\left(1+e^{(2 \varepsilon-2) u}\right), & \text { if } \frac{\sigma_{2}(W)}{\sigma_{1}(W)} \leq 2, \\ C\left(1+e^{\frac{(4 \varepsilon-4) u}{2-\varepsilon}}\right), & \text { if } \frac{\sigma_{2}(W)}{\sigma_{1}(W)} \geq 2 .\end{cases}
$$

Therefore, we have finished the proof of the theorem.

The same proof gives the local estimates for the elliptic Equation (2.13).

Theorem 3. Assume $n \geq 3$ and $\varepsilon \in\left[0, \varepsilon_{0}\right)$. Let $u$ be a solution of (2.13) in a geodesic ball $B_{r}$ for $r<r_{0}$, the injectivity radius of $M$. There is a constant $C$ depending only on $\left(B_{r}, g_{0}\right)$ (independent of $\varepsilon$ ) such that for any $\varepsilon \in\left[0, \varepsilon_{0}\right)$ and $x \in B_{r / 2}$

$$
|\nabla u|^{2}+\left|\nabla^{2} u\right| \leq C\left(1+\mathrm{e}^{-(2-2 \varepsilon) i n f_{x \in B_{r}} u(x)}\right) .
$$




\section{A Sobolev inequality}

The Sobolev inequality is a very important analytic tool in many problems arising from analysis and geometry. It plays a crucial role in the resolution of the Yamabe problem, which was solved completely by Yamabe [36], Trudinger [32], Aubin [1] and Schoen [29]. See various optimal Sobolev inequalities in [25]. In this section, we are interested in a similar type inequality for the class of a fully non-linear conformal quotient operators. In $[12,17$, 20,21 , the Sobolev inequality was generalized to the various fully non-linear operators.

In this section, we establish the Sobolev inequality relating $\int_{M} \sigma_{2}(g) d$ $\operatorname{vol}(g)$ and $\int_{M} \sigma_{1, \varepsilon}(g) d \operatorname{vol}(g)$ for a general manifold, which will be used in the next section.

Theorem 4. Let $\left(M, g_{0}\right)$ be a compact Riemannian manifold with $g_{0} \in \Gamma_{2}^{+}$ and the dimension $n>4$. Assume $\varepsilon \in[0,1 / 2]$. Then there exists a positive constant $C>0$ depending only on $\left(M, g_{0}\right)$ (and independent of $\varepsilon$ ) such that for any $C^{2}$ function $u$ with $\mathrm{e}^{-2 u} g_{0} \in \mathcal{C}_{1}\left(\left[g_{0}\right]\right)$ we have

$$
\int_{M} \sigma_{2}\left(\mathrm{e}^{-2 u} g_{0}\right) d \operatorname{vol}\left(\mathrm{e}^{-2 u} g_{0}\right) \geq C\left(\int_{M} \mathrm{e}^{2 \varepsilon u} \sigma_{1}\left(\mathrm{e}^{-2 u} g_{0}\right) d \operatorname{vol}\left(\mathrm{e}^{-2 u} g_{0}\right)\right)^{\frac{n-4}{n-2-2 \varepsilon}} \text {. }
$$

Equivalently, for such a function $u$ we have

$$
\begin{aligned}
& \int_{M} \mathrm{e}^{(4-n) u} \sigma_{2}\left(\nabla^{2} u+d u \otimes d u-\frac{|\nabla u|^{2}}{2} g_{0}+S_{g_{0}}\right) d \operatorname{vol}\left(g_{0}\right) \\
& \quad \geq C\left(\int_{M} \mathrm{e}^{(2+2 \varepsilon-n) u} \sigma_{1}\left(\nabla^{2} u+d u \otimes d u-\frac{|\nabla u|^{2}}{2} g_{0}+S_{g_{0}}\right) d \operatorname{vol}\left(g_{0}\right)\right)^{\frac{n-4}{n-2-2 \varepsilon}} .
\end{aligned}
$$

Proof. Let $g=\mathrm{e}^{-2 u} g_{0}$. We have shown in [11] the invariant $Y_{2,1}\left(\left[g_{0}\right]\right)>0$ and for any $\mathrm{e}^{-2 u} g_{0} \in \mathcal{C}_{1}\left(\left[g_{0}\right]\right)$

$$
\begin{aligned}
& \int_{M} \sigma_{2}\left(\mathrm{e}^{-2 u} g_{0}\right) d \operatorname{vol}\left(\mathrm{e}^{-2 u} g_{0}\right) \geq C_{1} \int_{M}|\nabla u|^{4} \mathrm{e}^{(4-n) u} d \operatorname{vol}\left(g_{0}\right) \\
&-C \int_{M} \mathrm{e}^{(4-n) u} d \operatorname{vol}\left(g_{0}\right) \\
& \int_{M} \sigma_{2}\left(\mathrm{e}^{-2 u} g_{0}\right) d \operatorname{vol}\left(\mathrm{e}^{-2 u} g_{0}\right) \geq C_{1} \int_{M} \mathrm{e}^{(4-n) u} d \operatorname{vol}\left(g_{0}\right),
\end{aligned}
$$


for some positive constants $C_{1}>0$ and $C>0$. Hence, we deduce

$$
\int_{M} \sigma_{2}\left(\mathrm{e}^{-2 u} g_{0}\right) d \operatorname{vol}\left(\mathrm{e}^{-2 u} g_{0}\right) \geq C \int_{M}|\nabla u|^{4} \mathrm{e}^{(4-n) u} d \operatorname{vol}\left(g_{0}\right) .
$$

It is easy to say that

$$
\begin{aligned}
\int_{M} & \mathrm{e}^{2 \varepsilon u} \sigma_{1}\left(\mathrm{e}^{-2 u} g_{0}\right) d \operatorname{vol}\left(\mathrm{e}^{-2 u} g_{0}\right) \\
= & \int\left(\Delta u-\frac{n-2}{2}|\nabla u|^{2}+\sigma_{1}\left(g_{0}\right)\right) \mathrm{e}^{(2+2 \varepsilon-n) u} d \operatorname{vol}\left(g_{0}\right) \\
= & \int\left(\frac{n-2-4 \varepsilon}{2}|\nabla u|^{2}+\sigma_{1}\left(g_{0}\right)\right) \mathrm{e}^{(2+2 \varepsilon-n) u} d \operatorname{vol}\left(g_{0}\right) \\
\leq & \frac{n-2-4 \varepsilon}{2}\left(\int|\nabla u|^{4} \mathrm{e}^{(4-n) u} d \operatorname{vol}\left(g_{0}\right) \int \mathrm{e}^{(4 \varepsilon-n) u} d \operatorname{vol}\left(g_{0}\right)\right)^{1 / 2} \\
& +\left(\sup \sigma_{1}\left(g_{0}\right)\right) \int \mathrm{e}^{(2+2 \varepsilon-n) u} d \operatorname{vol}\left(g_{0}\right) .
\end{aligned}
$$

Recall the definition of the conformal invariants [11]

$$
Y_{1}\left(\left[g_{0}\right]\right)=\inf _{g \in \mathcal{C}_{1}\left(\left[g_{0}\right]\right)} \frac{\int \sigma_{1}(g) d \operatorname{vol}(g)}{(\operatorname{vol}(g))^{\frac{n-2}{n}}}
$$

and

$$
\begin{aligned}
& \left(\int_{M} d \operatorname{vol}\left(\mathrm{e}^{-2 u} g_{0}\right)\right)^{\frac{n-4}{n}} \\
& \quad \leq\left(Y_{2,1}\left(\left[g_{0}\right]\right) Y_{1}\left(\left[g_{0}\right]\right)^{\frac{n-4}{n-2}}\right)^{-1} \int_{M} \sigma_{2}\left(\mathrm{e}^{-2 u} g_{0}\right) d \operatorname{vol}\left(e^{-2 u} g_{0}\right)
\end{aligned}
$$

By the Hölder inequality, we get for any $\alpha \in[0, n / 2]$

$$
\left(\int_{M} \mathrm{e}^{\alpha u} d \operatorname{vol}\left(\mathrm{e}^{-2 u} g_{0}\right)\right)^{\frac{n-4}{n-\alpha}} \leq C \int_{M} \sigma_{2}\left(\mathrm{e}^{-2 u} g_{0}\right) d \operatorname{vol}\left(\mathrm{e}^{-2 u} g_{0}\right)
$$


where $C$ is a positive constant independent of $\alpha$. Equations (4.5), (4.6) and (4.9) imply

$$
\begin{aligned}
\int_{M} \mathrm{e}^{2 \varepsilon u} \sigma_{1}\left(\mathrm{e}^{-2 u} g_{0}\right) d \operatorname{vol}\left(\mathrm{e}^{-2 u} g_{0}\right) \\
\leq C\left[\left(\int_{M} \sigma_{2}\left(\mathrm{e}^{-2 u} g_{0}\right) d \operatorname{vol}\left(\mathrm{e}^{-2 u} g_{0}\right)\right)\right)^{1 / 2} \\
\left.\times\left(\int_{M} \sigma_{2}\left(\mathrm{e}^{-2 u} g_{0}\right) d \operatorname{vol}\left(\mathrm{e}^{-2 u} g_{0}\right)\right)\right)^{\frac{n-4 \varepsilon}{2(n-4)}} \\
\left.\left.+\left(\int_{M} \sigma_{2}\left(\mathrm{e}^{-2 u} g_{0}\right) d \operatorname{vol}\left(\mathrm{e}^{-2 u} g_{0}\right)\right)\right)^{\frac{n-2-2 \varepsilon}{n-4}}\right] \\
\left.\leq C\left(\int_{M} \sigma_{2}\left(\mathrm{e}^{-2 u} g_{0}\right) d \operatorname{vol}\left(\mathrm{e}^{-2 u} g_{0}\right)\right)\right)^{\frac{n-2-2 \varepsilon}{n-4}}
\end{aligned}
$$

We finish the proof of theorem.

Remark 1. In [13], we proved the Sobolev inequality (4.1) in the cone $\mathcal{C}_{2}\left(\left[g_{0}\right]\right)$ when the conformal invariant $Y_{2,1}\left(\left[g_{0}\right]\right)>0$.

\section{Proof of Theorem 1 in the case $n \geq 5$}

Now we can prove that $Y_{\varepsilon}$ is achieved for any small $\varepsilon>0$.

Proposition 2. For $\varepsilon_{0}>\varepsilon>0$, flow (2.2) globally converges to a solution of (2.12). As a direct application, $Y_{\varepsilon}$ is achieved by a function $u_{\varepsilon}$ satisfying (2.12).

Proof. We divide the proof into three steps.

Step 1. For a fixed small number $\varepsilon>0$, the solution $u$ of flow (2.2) has a uniform $C^{0}$ bound, which is independent of $t$.

The proof use the optimality of the local estimate (3.1). First all, since flow (2.2) does not increase $\mathcal{F}_{2}, \mathcal{F}_{2}(g)$ is bounded from above along the flow. By (4.8), we know that $\int_{M} d \operatorname{vol}(g)$ is bounded from above. From the Hölder inequality, $V_{\varepsilon}(g)=\int_{M} \mathrm{e}^{2 \varepsilon u} d \operatorname{vol}(g)$ is also bounded.

Let $T^{*} \in(0, \infty]$ be the maximum of the existence of the flow. For any $T \in\left[0, T^{*}\right)$, set

$$
m(T)=\min _{(x, s) \in M \times[0, T]} u(x, s) .
$$


We show that there is a constant $C_{0}>0$ independent of $T$ (depending on $\varepsilon)$ such that

$$
\inf _{T \in\left[0, T^{*}\right)} m(T)>-C_{0}
$$

We assume by contradiction that $\inf _{t \in\left[0, T^{*}\right)} m(t)=-\infty$. Let $T_{i}$ be a sequence tending to $T^{*}$ with $m\left(T_{i}\right) \rightarrow-\infty$ as $i \rightarrow \infty$. Let $\left(x_{i}, t_{i}\right) \in M \times$ $\left[0, T_{i}\right]$ with $u\left(x_{i}, t_{i}\right)=m\left(T_{i}\right)$. Fix $\delta \in\left(\frac{2}{5}, \frac{1}{2}\right)$, we consider $r_{i}=\frac{\varepsilon}{2}\left|m\left(T_{i}\right)\right|$ $\mathrm{e}^{(1-\delta \varepsilon) m\left(T_{i}\right)}$. Clearly, we have $r_{i} \rightarrow 0$. It follows from Theorem 2 that for sufficiently large $i$ and for any $x \in B_{r_{i}}\left(x_{i}\right)$

$$
\begin{aligned}
u\left(x, t_{i}\right) & \leq m\left(T_{i}\right)+\left(\sup _{B_{r_{i}}\left(x_{i}\right)}|\nabla u|\right) r_{i} \\
& \leq m\left(T_{i}\right)+C \mathrm{e}^{\left(\frac{\varepsilon}{2-\varepsilon}-1\right) m\left(T_{i}\right)} \frac{\varepsilon}{2}\left|m\left(T_{i}\right)\right| \mathrm{e}^{(1-\delta \varepsilon) m\left(T_{i}\right)} \\
& =m\left(T_{i}\right)+C \frac{\varepsilon}{2}\left|m\left(T_{i}\right)\right| \mathrm{e}^{\varepsilon\left(\frac{1}{2-\varepsilon}-\delta\right) m\left(T_{i}\right)} \\
& \leq(1-\kappa) m\left(T_{i}\right)
\end{aligned}
$$

for some $\kappa \in\left(0,\left(\delta-\frac{2}{n}\right) \varepsilon\right)$. Note that $\delta-\frac{2}{n}>0$, for $n \geq 5$. Therefore, we obtain

$$
\begin{aligned}
\int_{B\left(x_{i}, r_{i}\right)} \mathrm{e}^{2 \varepsilon u} d \operatorname{vol}(g) & \geq \int_{B\left(x_{i}, r_{i}\right)} \mathrm{e}^{(2 \varepsilon-n) m\left(T_{i}\right)(1-\kappa)} d \operatorname{vol}\left(g_{0}\right) \\
& \geq C \mathrm{e}^{(2 \varepsilon-n) m\left(T_{i}\right)(1-\kappa)} r_{i}^{n} \\
& \geq C\left(\frac{\left|m\left(T_{i}\right)\right| \varepsilon}{2}\right)^{n} \rightarrow \infty
\end{aligned}
$$

where we have used $n \geq 5$. Hence, this fact contradicts the boundedness of $V_{\varepsilon}$. This proves the claim. This claim, together with the local estimates and the fact $\mathcal{F}_{1, \varepsilon}$ is preserved along the flow, implies that $\|u(t)\|_{C^{2}}$ has a unform bound.

Step 2. We prove a crucial fact that the flow preserves $\Gamma_{1}^{+}$metrics. More precisely, we have the following result:

Lemma 5. There is a constant $C_{0}>0$, independent of $T \in\left[0, T^{*}\right)$ such that $\sigma_{1}(g(x, t))>C_{0}$ for any $t \in[0, T]$ and $x \in M$. 
Proof of Lemma 5. From the Sobolev inequality and Lemma 2, the function $r_{\varepsilon}(g(t))$ is bounded from below and from above by the positive constants. Recall

$$
\begin{gathered}
\nu=\varepsilon\left(|\nabla u|^{2}+2 \sigma_{1}\left(g_{0}\right) /(n-2-4 \varepsilon)\right) \\
W=\left(w_{i j}\right)=\left(\nabla_{i j}^{2} u+u_{i} u_{j}-\frac{|\nabla u|^{2}}{2}\left(g_{0}\right)_{i j}+\left(S_{g_{0}}\right)_{i j}\right), \\
K=r_{\varepsilon}(g(t)) \mathrm{e}^{(2 \varepsilon-2) u}, K_{1}=K\left(1+\frac{\nu}{\sigma_{1}(W)}\right)
\end{gathered}
$$

and

$$
F_{\varepsilon}(W, \nu, u, t)=h_{\varepsilon}\left(\frac{\sigma_{2}(W)}{\sigma_{1}(W)}\right)-h_{\varepsilon}\left(K_{1}\right) .
$$

Let us consider a function $H_{\varepsilon}: M \times[0, T]$ defined by

$$
\begin{aligned}
H_{\varepsilon} & :=F_{\varepsilon}-\mathrm{e}^{-u} \\
& =u_{t}-s_{\varepsilon}(g)-\mathrm{e}^{-u},
\end{aligned}
$$

where $u_{t}$ denotes the derivative of $u$ with respect to $t$. Without loss of generality, we assume that the minimum of $H_{\varepsilon}$ is achieved at $\left(x_{0}, t_{0}\right) \in M \times$ $(0, T]$ and at $\left(x_{0}, t_{0}\right)$

$$
\frac{\sigma_{2}(W)}{\sigma_{1}(W)}<1
$$

Recall that $h_{\varepsilon}(t)=t$ for $t<1$. Hence, in a small neighborhood of $\left(x_{0}, t_{0}\right)$

$$
H_{\varepsilon}=\frac{\sigma_{2}(W)}{\sigma_{1}(W)}-h_{\varepsilon}\left(K_{1}\right)-\mathrm{e}^{-u}
$$

Let us use $O(1)$ denote terms with a uniform bound (perhaps depending on $\varepsilon)$. Using $\frac{d r_{\varepsilon}(g)}{d t} \leq 0$, we have near $\left(x_{0}, t_{0}\right)$

$$
\begin{aligned}
\frac{d}{d t} H_{\varepsilon} \geq & \operatorname{tr}\left(A \nabla_{g}^{2}\left(H_{\varepsilon}+\mathrm{e}^{-u}\right)\right)-h_{\varepsilon}^{\prime}\left(K_{1}\right) K \frac{2 \varepsilon\left\langle\nabla_{g_{0}} u, \nabla_{g_{0}}\left(H_{\varepsilon}+\mathrm{e}^{-u}\right)\right\rangle_{g_{0}}}{\sigma_{1}(W)} \\
& +k_{\varepsilon}(x, t) u_{t}
\end{aligned}
$$

where

$$
A:=\frac{\left(\sigma_{1}^{2}(W)-\sigma_{2}(W)\right) I-\sigma_{1}(W) W}{\sigma_{1}^{2}(W)}+h_{\varepsilon}^{\prime}\left(K_{1}\right) \frac{K \nu I}{\sigma_{1}^{2}(W)}
$$

is positive definite and

$$
k_{\varepsilon}(x, t):=\mathrm{e}^{-u}+(2-2 \varepsilon) h_{\varepsilon}^{\prime}\left(K_{1}\right) K_{1}
$$


is a positive function and $I$ denotes the identity matrix. To simplify the notations, we drop the index $\varepsilon$ as before. We prove first, there is a constant $C_{2}>0$, independent of $T \in\left[0, T^{*}\right)$ such that $\sigma_{1}\left(g\left(x_{0}, t_{0}\right)\right)>C_{2}$. Since $\left(x_{0}, t_{0}\right)$ is the minimum of $H$ in $M \times[0, T]$, at this point, we have $\frac{d H}{d t} \leq 0$, $H_{l}=0 \forall l$ and $\left(H_{i j}\right)$ is non-negative definite. Note that

$$
\left(\nabla_{g}^{2}\right)_{i j} H=H_{i j}+u_{i} H_{j}+u_{j} H_{i}-\sum_{l} u_{l} H_{l} \delta_{i j}=H_{i j}
$$

at $\left(x_{0}, t_{0}\right)$, where $H_{j}$ and $H_{i j}$ are the first and second derivatives with respect to the back-ground metric $g_{0}$. From the positivity of $A$ and (5.2), we have

$$
\begin{aligned}
0 & \geq H_{t}-\sum_{i, j} A^{i j} H_{i j} \\
& \geq \sum_{i, j} A^{i j}\left\{\left(\mathrm{e}^{-u}\right)_{i j}+u_{i}\left(\mathrm{e}^{-u}\right)_{j}+u_{j}\left(\mathrm{e}^{-u}\right)_{i}-\sum_{l} u_{l}\left(\mathrm{e}^{-u}\right)_{l} \delta_{i j}\right\}+k(x, t) u_{t} \\
& =\mathrm{e}^{-u} \sum_{i, j} A^{i j}\left\{-w_{i j}+S\left(g_{0}\right)_{i j}+\frac{1}{2}|\nabla u|^{2} \delta_{i j}\right\}+k(x, t) u_{t} \\
& \geq \mathrm{e}^{-u} \sum_{i, j} A^{i j}\left(-w_{i j}+S\left(g_{0}\right)_{i j}\right)+k(x, t) u_{t} .
\end{aligned}
$$

Here we have

$$
\sum_{i, j} A^{i j} w_{i j}=\frac{\sigma_{2}(W)}{\sigma_{1}(W)}+h^{\prime}\left(K_{1}\right) \frac{K \nu}{\sigma_{1}(W)}
$$

On the other hand, we have

$$
\begin{aligned}
\sum_{i, j} A^{i j} S\left(g_{0}\right)_{i j}= & \frac{\sigma_{1}\left(g_{0}\right)\left(\sigma_{1}^{2}(W)-\sigma_{2}(W)\right)}{\sigma_{1}^{2}(W)}-\frac{1}{\sigma_{1}(W)} \sum_{i, j} w^{i j} S\left(g_{0}\right)_{i j} \\
& +h^{\prime}\left(K_{1}\right) \frac{K \nu \sigma_{1}\left(g_{0}\right)}{\sigma_{1}^{2}(W)} \\
= & -\frac{\sigma_{2}(W) \sigma_{1}\left(g_{0}\right)}{\sigma_{1}^{2}(W)}+h^{\prime}\left(K_{1}\right) \frac{K \nu \sigma_{1}\left(g_{0}\right)}{\sigma_{1}^{2}(W)} \\
& -\frac{1}{\sigma_{1}(W)} \sum_{i, j} w^{i j} S\left(g_{0}\right)_{i j}+\sigma_{1}\left(g_{0}\right) .
\end{aligned}
$$


As $W$ is bounded, we deduce that

$$
\begin{gathered}
\sum_{i, j} A^{i j}\left(-w_{i j}+S\left(g_{0}\right)_{i j}\right) \\
=\left(h^{\prime}\left(K_{1}\right) \frac{K \nu \sigma_{1}\left(g_{0}\right)}{\sigma_{1}^{2}(W)}-\frac{\sigma_{2}(W) \sigma_{1}\left(g_{0}\right)}{\sigma_{1}^{2}(W)}+O\left(\frac{1}{\sigma_{1}(W)}\right)+O(1)\right) \\
k\left(x_{0}, t_{0}\right)=(2-2 \varepsilon) h^{\prime}\left(K_{1}\right) \frac{K \nu}{\sigma_{1}(W)}+O(1) \\
u_{t}\left(x_{0}, t_{0}\right)=H\left(x_{0}, t_{0}\right)+s_{\varepsilon}\left(g\left(t_{0}\right)\right)+\mathrm{e}^{-u\left(x_{0}, t_{0}\right)} \geq H\left(x_{0}, t_{0}\right)+O(1) \\
=\frac{\sigma_{2}(W)}{\sigma_{1}(W)}-h\left(K_{1}\right)+O(1)
\end{gathered}
$$

We divide the proof into two cases.

Case 1: $\sigma_{2}(W) \geq 0$.

It is clear

$$
0 \leq \sigma_{2}(W) \leq \frac{1}{2} \sigma_{1}^{2}(W)
$$

Thus,

$$
\begin{aligned}
0 \geq & \mathrm{e}^{-u} h^{\prime}\left(K_{1}\right) \frac{K \nu \sigma_{1}\left(g_{0}\right)}{\sigma_{1}^{2}(W)}-(2-2 \varepsilon) h\left(K_{1}\right) h^{\prime}\left(K_{1}\right) \frac{K \nu}{\sigma_{1}(W)} \\
& +O(1)+O\left(\frac{1}{\sigma_{1}(W)}\right) .
\end{aligned}
$$

Assume that $\frac{1}{\sigma_{1}(W)}$ is sufficiently large. Then

$$
h^{\prime}\left(K_{1}\right) \frac{K \nu \sigma_{1}\left(g_{0}\right)}{\sigma_{1}^{2}(W)} \geq \frac{C_{3}}{\sigma_{1}^{2-\varepsilon / 2}(W)}
$$

and

$$
(2-2 \varepsilon) h\left(K_{1}\right) h^{\prime}\left(K_{1}\right) \frac{K \nu}{\sigma_{1}(W)} \leq \frac{C_{4}}{\sigma_{1}^{2-\varepsilon}(W)}
$$

for some positive constants $C_{3}$ and $C_{4}$ independent of $T$. This implies boundness of $\sigma_{1}(W)$ at the point $\left(x_{0}, t_{0}\right)$ from below by some positive constant independent of $T$. 
Case 2: $\sigma_{2}(W)<0$.

In this case, $(5.8)$ holds also since $-\frac{\sigma_{1}\left(g_{0}\right) \sigma_{2}(W)}{\sigma_{1}^{2}(W)}+k\left(x_{0}, t_{0}\right) \frac{\sigma_{2}(W)}{\sigma_{1}(W)}>0$, provided $\frac{1}{\sigma_{1}(W)}$ is sufficiently large. Hence, the desired result yields.

Now for any $(x, t) \in M \times[0, T]$ we have

$$
H(x, t) \geq H\left(x_{0}, t_{0}\right)=O(1)
$$

so that

$$
h\left(\frac{\sigma_{2}(W)}{\sigma_{1}(W)}\right)(x, t)-h\left(K_{1}\right)(x, t) \geq O(1) .
$$

Therefore, we infer

$$
-h\left(r_{\varepsilon}(g(t)) \mathrm{e}^{(2 \varepsilon-2) u}\left(1+\frac{\nu}{\sigma_{1}(W)}\right)\right)(x, t) \geq O(1),
$$

since we have always

$$
\left\{\begin{array}{l}
h\left(\frac{\sigma_{2}(W)}{\sigma_{1}(W)}\right)(x, t)=\frac{\sigma_{2}(W)}{\sigma_{1}(W)}(x, t)<0, \quad \text { if } \sigma_{2}(W)(x, t)<0 \\
\frac{\sigma_{2}(W)}{\sigma_{1}(W)}(x, t) \leq \frac{1}{2} \sigma_{1}(W)(x, t) \leq O(1), \quad \text { if } \sigma_{2}(W)(x, t) \geq 0
\end{array}\right.
$$

Finally, $K_{1}(x, t)$ is bounded from above and yields that there exists $C_{0}>0$ independent of $T$ such that $\sigma_{1}(W)(x, t)>C_{0}$. Therefore, lemma is proved.

Proof of Proposition 2 (continued). Step 3: Now we can finish the proof of Proposition 2. From Step 2, we know that the flow is uniformly parabolic. In view of Step 1, Krylov's theory implies $u(t)$ has a uniform $C^{2, \alpha}$ bound. Hence, $T^{*}=\infty$. One can also show that $u(t)$ globally converges to $u(\infty)$, which clearly is a solution of (2.12) for $\varepsilon_{0}>\varepsilon>0$ (see [31]). From the local estimates, the set of solutions of (2.12) for $c=1$ with the uniform bounded energy functional $\mathcal{F}_{2}$ is bounded in $C^{2}$ norm. Since (2.13) is concave in $W$, from the Evans-Krylov theory, this set is compact in $C^{2, \alpha}$ norm. Now it is easy to show that $Y_{\varepsilon}$ is achieved.

Proof of Theorem 1 in the case $n \geq 5$. By Proposition 2, for small $\varepsilon>0$, we have a solution $u_{\varepsilon}$ of $(2.12)$ that has $\tilde{\mathcal{F}}_{2, \varepsilon}\left(u_{\varepsilon}\right)=Y_{\varepsilon}$. It is easy to show that

$$
\lim _{\varepsilon \rightarrow 0} Y_{\varepsilon}=\lim _{\varepsilon \rightarrow 0} \tilde{Y}_{\varepsilon}=Y_{2,1}
$$


If $\lim _{\varepsilon \rightarrow 0} \min _{x \in M} u_{\varepsilon}(x)>-\infty$, then local estimates imply that $u_{\varepsilon}$ (taking a subsequence) converges in $C^{2, \alpha}$ to $u$, which is a solution of (1.3). We are done.

If $\lim _{\varepsilon \rightarrow 0} \min _{x \in M} u_{\varepsilon}(x)=-\infty$, we can use the local estimates and the classification of solutions of (2.14) in the standard sphere to get a contradiction to the facts $\tilde{Y}_{2,1}\left(\left[g_{0}\right]\right) \leq \tilde{Y}_{2,1}\left(S^{n}\right)$ and equality holds if only if $\left(M,\left[g_{0}\right]\right)$ is the standard sphere. This so-called the blow-up analysis for this class of fully non-linear conformal equations becomes more or less standard. Here, we leave the proof to the interested reader.

Now $Y_{2,1}\left(\left[g_{0}\right]\right)$ is achieved by some $g \in \mathcal{C}_{1}\left(\left[g_{0}\right]\right)$, which solves (2.14). Hence, $g \in \mathcal{C}_{2}\left(\left[g_{0}\right]\right)$ and yields that $Y_{2,1}\left(\left[g_{0}\right]\right)=\tilde{Y}_{2,1}\left(\left[g_{0}\right]\right)$.

\section{Proof of Theorem 1 in the case $n=3$}

Now we want to consider the existence of the following equation:

$$
F_{\varepsilon}(g)=\frac{\sigma_{2}(g)-\varepsilon e^{4 u}}{\sigma_{1}(g)}=\mathrm{constant}
$$

with $g=\mathrm{e}^{-2 u} g_{0}$ for $\varepsilon>0$ a positive number. In this paper, we will choose $\varepsilon$ as a small positive constant. Following $[12,13,20]$, we will introduce a suitable Yamabe-type flow to study Equation (6.1).

For any $\varepsilon \in(0,+\infty)$ and for $g=\mathrm{e}^{-2 u} g_{0}$, consider the following perturbed functional:

$$
\mathcal{E}_{\varepsilon}(g):= \begin{cases}\frac{2}{n-4} \int_{M}\left(\sigma_{2}(g)-\varepsilon \mathrm{e}^{4 u}\right) d \operatorname{vol}(g), & \text { if } n \neq 4 \\ -\int_{0}^{1} \int_{M}\left(\sigma_{2}\left(g_{t}\right)-2 \varepsilon \mathrm{e}^{4 t u}\right) u d \operatorname{vol}\left(g_{t}\right) d t, & \text { if } n=4\end{cases}
$$

where $g_{t}=\mathrm{e}^{-2 t u} g_{0}$. When $\varepsilon=0$, the functional was considered in $[4,5,33]$. Recall

$$
\mathcal{F}_{1}(g)=\int_{M} \sigma_{1}(g) d \operatorname{vol}(g) \quad \text { and } \quad \mathcal{F}_{2}(g)=\int_{M} \sigma_{2}(g) d \operatorname{vol}(g)
$$

From the variational formula given in $[4,5,33]$, we have

$$
\frac{d}{d t} \mathcal{E}_{\varepsilon}(g)=\int\left(\sigma_{2}(g)-\varepsilon \mathrm{e}^{4 u}\right) g^{-1} \cdot \frac{d}{d t} g d \operatorname{vol}(g)
$$


and

$$
\frac{d}{d t} \mathcal{F}_{1}(g)=\frac{n-2}{2} \int \sigma_{1}(g) g^{-1} \cdot \frac{d}{d t} g d \operatorname{vol}(g) .
$$

Now we introduce a Yamabe-type flow, which non-increases $\mathcal{E}_{\varepsilon}$ and preserves $\mathcal{F}_{1}$.

$$
\frac{d u}{d t}=-\frac{1}{2} g^{-1} \frac{d}{d t} g=\mathrm{e}^{-2 u} \frac{\sigma_{2}(g)-\varepsilon \mathrm{e}^{4 u}}{\sigma_{1}(g)}-r_{\varepsilon}(g) \mathrm{e}^{-2 u}+s_{\varepsilon}(g),
$$

where $r_{\varepsilon}(g)$ and $s_{\varepsilon}(g)$ are space constants, given by

$$
r_{\varepsilon}(g):=\frac{\mathcal{F}_{2}(g)-\int_{M} \varepsilon \mathrm{e}^{4 u} d \operatorname{vol}_{g}}{\mathcal{F}_{1}(g)}
$$

and

$$
\int_{M} \sigma_{1}(g)\left\{\mathrm{e}^{-2 u} \frac{\sigma_{2}(g)-\varepsilon \mathrm{e}^{4 u}}{\sigma_{1}(g)}-r_{\varepsilon}(g) \mathrm{e}^{-2 u}+s_{\varepsilon}(g)\right\} d \operatorname{vol}(g)=0 .
$$

We collect some basic facts proved in [11].

Lemma 6. Assume flow (6.4) stays in the cone $\Gamma_{1}^{+}$. Then the flow preserves $\mathcal{F}_{1}$ and non-increases $\mathcal{E}_{\varepsilon}$. Hence when $n \geq 4$, then $r_{\varepsilon}$ is non-increasing along the flow, and when $n=3$, then $r_{\varepsilon}$ is non-decreasing along the flow.

Given $\varepsilon>0$, assume $g_{0} \in \mathcal{C}_{1}\left(\left[g_{0}\right]\right)$. By Lemma 3, (6.4) is parabolic. By the standard implicit function theorem we have the short-time existence result. Let $T^{*} \in(0, \infty]$, so that $\left[0, T^{*}\right)$ is the maximum interval for the existence of the flow $g(t) \in \Gamma_{1}^{+}$.

Proposition 3. Assume that $n \geq 3, \varepsilon>0$ and $g_{0} \in \Gamma_{1}^{+}$. Let $u$ be a solution of (6.4) in a geodesic ball $B_{R} \times[0, T]$ for $T<T^{*}$ and $R<\tau_{0}$, the injectivity radius of $M$.

(1) Assume that $\forall t \in[0, T]$, there holds

$$
r_{\varepsilon}(t) \leq 0
$$

Then there is a constant $C$ depending only on $\left(B_{R}, g_{0}\right)$ (independent of $\varepsilon$ and $T$ ) such that for any $(x, t) \in B_{R / 2} \times[0, T]$

$$
|\nabla u|^{2}+\left|\nabla^{2} u\right| \leq C
$$


(2) Assume that $\forall t \in[0, T]$, there holds

$$
r_{\varepsilon}(t)>0
$$

Then there is a constant $C$ depending only on $\left(B_{R}, g_{0}\right)$ (independent of $\varepsilon$ and $T)$ such that for any $(x, t) \in B_{R / 2} \times[0, T]$

$$
|\nabla u|^{2}+\left|\nabla^{2} u\right| \leq C\left(1+\sup _{t \in[0, T]} r_{\varepsilon}(t) \times \mathrm{e}^{-2 \inf _{(x, t) \in B_{R} \times[0, T]} u(x, t)}\right)
$$

Now we define

$$
a_{\varepsilon}:=\inf _{g \in \mathcal{C}_{1}\left(\left[g_{0}\right]\right)} \frac{\mathcal{E}_{\varepsilon}(g)}{\left(\int_{M} \sigma_{1}(g) d \operatorname{vol}(g)\right)^{\frac{n-4}{n-2}}} \text {,if } n \neq 4 .
$$

If $a_{\varepsilon}$ is achieved by a metric $g=\mathrm{e}^{-2 u} g_{0}$, the $g$ satisfies

$$
\frac{\sigma_{2}(g)-\varepsilon \mathrm{e}^{4 u}}{\sigma_{1}(g)}=\kappa
$$

for some constant $\kappa$. Equivalently, we will consider the energy functional $\mathcal{E}_{\varepsilon}$ on the normalized cone $\tilde{\mathcal{C}}_{1}\left(\left[g_{0}\right]\right)$

$$
\tilde{\mathcal{C}}_{1}\left(\left[g_{0}\right]\right):=\left\{g \in \mathcal{C}_{1}\left(\left[g_{0}\right]\right) \mid \int_{M} \sigma_{1}(g) d \operatorname{vol}(g)=1\right\} .
$$

Using the same arguments as in Proposition 3, we have the following local estimate.

Proposition 4. Assume that $n \geq 3, \varepsilon>0$ and $g_{0} \in \Gamma_{1}^{+}$. Let $u$ be a solution of (6.10) in a geodesic ball $B_{R} \times[0, T]$ for $T<T^{*}$ and $R<\tau_{0}$, the injectivity radius of $M$.

(1) Assume

$$
\kappa \leq 0
$$

Then there is a constant $C$ depending only on $\left(B_{R}, g_{0}\right)$ (independent of $\varepsilon$ and $T$ ) such that for any $(x, t) \in B_{R / 2} \times[0, T]$

$$
|\nabla u|^{2}+\left|\nabla^{2} u\right| \leq C
$$


(2) Assume

$$
\kappa>0
$$

Then there is a constant $C$ depending only on $\left(B_{R}, g_{0}\right)$ (independent of $\varepsilon$ and $T$ ) such that for any $(x, t) \in B_{R / 2} \times[0, T]$

$$
|\nabla u|^{2}+\left|\nabla^{2} u\right| \leq C\left(1+\kappa \times \mathrm{e}^{-2 \inf _{(x, t) \in B_{R} \times[0, T]} u(x, t)}\right) .
$$

Now we consider $n=3$ and can prove that $a_{\varepsilon}$ is achieved for any small $\varepsilon>0$.

Proposition 5. Assume $g_{0} \in \Gamma_{1}^{+}$and $n=3$. For $\frac{1}{2}>\varepsilon>0$, flow (6.4) globally converges to a solution of (6.10). As a direct application, $a_{\varepsilon}$ is achieved by a function $u_{\varepsilon}$ satisfying (6.10) for $\kappa>0$, provided $\varepsilon$ is sufficiently small.

Proof. We divide the proof into three steps.

Step 1. There is a constant $C_{0}>0$, independent of $T \in\left[0, T^{*}\right)$ such that

$$
\|u(t)\|_{C^{2}} \leq C_{0}
$$

Claim. There is a constant $C>0$, independent of $T \in\left[0, T^{*}\right)$ such that

$$
\int_{M} \mathrm{e}^{4 u(t)} d \operatorname{vol}(g(t)) \leq C
$$

Without loss of generality, we can suppose $\mathcal{F}_{1}(g(t)) \equiv 1$. Thus, we obtain

$$
\varepsilon \int_{M} \mathrm{e}^{4 u(t)} d \operatorname{vol}(g(t))=\mathcal{F}_{2}(g(t))-r_{\varepsilon}(g(t)) \leq \mathcal{F}_{2}(g(t)) \leq Y_{2,1}\left(\left[g_{0}\right]\right)
$$

Thus, we prove the claim. As in [11], we have for all $g \in \mathcal{C}_{1}\left(\left[g_{0}\right]\right)$

$$
\int \sigma_{2}(g) d \operatorname{vol}(g) \leq-\frac{1}{16} \int|\nabla u|_{g_{0}}^{4} e^{4 u} d \operatorname{vol}(g)+c \int e^{4 u} d \operatorname{vol}(g),
$$

for some positive constant $c>0$. Recall that $Y_{2,1}\left(\left[g_{0}\right]\right)$ is finite and $\int \sigma_{2}(g(t))$ $d \operatorname{vol}(g(t)) \geq 0$, provided $r_{\varepsilon}(g(0))>0$, since $r_{\varepsilon}(g(t))$ is non-decreasing. Thus, 
we infer $\forall t \in\left[0, T^{*}\right)$

$$
\begin{aligned}
256 \int\left|\nabla e^{\frac{u}{4}}\right|_{g_{0}}^{4} d \operatorname{vol}\left(g_{0}\right) & =\int|\nabla u|_{g_{0}}^{4} \mathrm{e}^{4 u} d \operatorname{vol}(g) \leq c \int \mathrm{e}^{4 u} d \operatorname{vol}(g) \\
& \leq c \int\left(\mathrm{e}^{u / 4}\right)^{4} d \operatorname{vol}\left(g_{0}\right)
\end{aligned}
$$

which implies by the Sobolev's embedding theorem for all $x, y \in M$

$$
\left|\mathrm{e}^{u(x, t) / 4}-\mathrm{e}^{u(y, t) / 4}\right| \leq c\left(\int \mathrm{e}^{4 u} d \operatorname{vol}(g)\right)^{1 / 4}\left(d_{g_{0}}(x, y)\right)^{1 / 4} \leq c\left(d_{g_{0}}(x, y)\right)^{1 / 4},
$$

where $d_{g_{0}}(x, y)$ is the distance between $x$ and $y$ with respect to the metric $g_{0}$. Set

$$
\beta(t):=\mathrm{e}^{\min _{M} u(\cdot, t)}=\mathrm{e}^{u\left(x_{t}, t\right)}
$$

and

$$
\tilde{\beta}(t):=\mathrm{e}^{\max _{M} u(\cdot, t)}=\mathrm{e}^{u\left(\tilde{x}_{t}, t\right)}
$$

for some $x_{t}, \tilde{x}_{t} \in M$. It follows from (6.19) that for any $y \in M$

$$
\mathrm{e}^{-u(y, t) / 4} \geq\left(\beta(t)^{1 / 4}+c\left(d_{g_{0}}(x, y)\right)^{1 / 4}\right)^{-1}
$$

which implies

$$
\begin{aligned}
\operatorname{Vol}(g(t)) & =\int \mathrm{e}^{-3 u(t)} d \operatorname{vol}\left(g_{0}\right) \geq \int\left(\beta(t)^{1 / 4}+c\left(d_{g_{0}}(x, y)\right)^{1 / 4}\right)^{-12} d \operatorname{vol}\left(g_{0}\right) \\
& \geq c \int_{0}^{R}\left(\beta(t)^{1 / 4}+c r^{1 / 4}\right)^{-12} r^{2} d r \geq-c \ln \beta(t),
\end{aligned}
$$

provided $\beta(t)<1 / 2$. On the other hand, we have always $\operatorname{Vol}(g(t)) \leq\left(Y_{1}\left(\left[g_{0}\right]\right)\right.$ $\left.\mathcal{F}_{1}(g(t))\right)^{3}$. Thus the lower boundness of $u(x, t)$ yields. Together with the local estimates and the fact $\mathcal{F}_{1}$ is preserved along the flow, this implies that $\|u(t)\|_{C^{2}}$ has a uniform bound.

Step 2. The flow preserves the positivity of the scalar curvature.

Proposition 6. There is a constant $C_{0}>0$, independent of $T \in\left[0, T^{*}\right)$ such that $\sigma_{1}(g(t))>C_{0}$ for any $t \in[0, T]$. 
Proof. Recall

$$
W=\left(w_{i j}\right)=\left(\nabla_{i j}^{2} u+u_{i} u_{j}-\frac{|\nabla u|^{2}}{2}\left(g_{0}\right)_{i j}+\left(S_{g_{0}}\right)_{i j}\right) .
$$

Set

$$
F_{\varepsilon}(W)=\frac{\sigma_{2}(W)-\varepsilon}{\sigma_{1}(W)}-\alpha \mathrm{e}^{-2 u} .
$$

where $\alpha=2 Y_{2,1}\left(\left[g_{0}\right]\right)$. Thus, $F_{\varepsilon}=u_{t}+\left(r_{\varepsilon}(g(t))-\alpha\right) \mathrm{e}^{-2 u}-s_{\varepsilon}(g(t))$. Without loss of generality, we assume that the minimum of $F_{\varepsilon}$ is achieved at $\left(x_{0}, t_{0}\right) \in M \times(0, T]$. Let us use $O(1)$ denote terms with a uniform bound with respect to $t$ (perhaps depending on $\varepsilon$ ). It is clear that $s_{\varepsilon}(g(t))$ and $r_{\varepsilon}(g(t))$ are bounded. Near $\left(x_{0}, t_{0}\right)$, we have

$$
\frac{d}{d t} F_{\varepsilon}=\sum_{i j} A^{i j}\left[\left(\nabla_{g}^{2}\left(F_{\varepsilon}\right)\right)_{i j}-\left(r_{\varepsilon}(g)-\alpha\right)\left(\nabla_{g}^{2}\left(\mathrm{e}^{-2 u}\right)\right)_{i j}\right]+2 \alpha \mathrm{e}^{-2 u} u_{t}
$$

where

$$
A^{i j}:=\frac{\left(\sigma_{1}^{2}(W)-\sigma_{2}(W)+\varepsilon\right) \delta^{i j}-\sigma_{1}(W) W^{i j}}{\sigma_{1}^{2}(W)}
$$

is positive definite. To simplify the notations, we drop the index $\varepsilon$ as before. Since $\left(x_{0}, t_{0}\right)$ is the minimum of $F$ in $M \times[0, T]$, at this point, we have $\frac{d F}{d t} \leq 0, F_{l}=0 \forall l$ and $\left(F_{i j}\right)$ is non-negative definite. Note that

$$
\left(\nabla_{g}^{2}\right)_{i j} F=F_{i j}+u_{i} F_{j}+u_{j} F_{i}-\sum_{l} u_{l} F_{l} \delta_{i j}=F_{i j}
$$

at $\left(x_{0}, t_{0}\right)$, where $F_{j}$ and $F_{i j}$ are the first and second derivatives with respect to the back-ground metric $g_{0}$. From the positivity of $A$ and (6.24), we have

$$
\begin{aligned}
(6.25)- & 2 \alpha \mathrm{e}^{-2 u} u_{t} \\
& =-2 \alpha \mathrm{e}^{-2 u} \frac{\sigma_{2}(W)-\varepsilon}{\sigma_{1}(W)}+O(1) \\
& \geq F_{t}-\sum_{i, j} A^{i j} F_{i j}-2 \alpha \mathrm{e}^{-2 u} u_{t} \\
& \geq\left(\alpha-r_{\varepsilon}(g)\right) \sum_{i, j} A^{i j}\left\{\left(\mathrm{e}^{-2 u}\right)_{i j}+u_{i}\left(\mathrm{e}^{-2 u}\right)_{j}\right.
\end{aligned}
$$




$$
\begin{aligned}
& \left.+u_{j}\left(\mathrm{e}^{-2 u}\right)_{i}-\sum_{l} u_{l}\left(\mathrm{e}^{-2 u}\right)_{l} \delta_{i j}\right\} \\
& =\left(\alpha-r_{\varepsilon}(g)\right) \mathrm{e}^{-2 u} \sum_{i, j} A^{i j}\left\{-2 w_{i j}+2 u_{i} u_{j}+2 S\left(g_{0}\right)_{i j}+|\nabla u|^{2} \delta_{i j}\right\} \\
& \geq\left(\alpha-r_{\varepsilon}(g)\right) \mathrm{e}^{-2 u}\left[\left(\frac{-2 \sigma_{2}(W)-2 \varepsilon}{\sigma_{1}(W)}\right)\right. \\
& \left.+\sum_{i, j} A^{i j}\left(2 u_{i} u_{j}+2 S\left(g_{0}\right)_{i j}+|\nabla u|^{2} \delta_{i j}\right)\right] .
\end{aligned}
$$

Here we have used $\sum_{i, j} A^{i j} w_{i j}=\frac{\sigma_{2}(W)+\varepsilon}{\sigma_{1}(W)}$. On the other hand, we have

$$
\begin{aligned}
\sum_{i, j} A^{i j} S\left(g_{0}\right)_{i j}= & \frac{\left(\sigma_{1}^{2}(W)-\sigma_{2}(W)\right) \sigma_{1}\left(g_{0}\right)}{\sigma_{1}^{2}(W)} \\
& -\frac{1}{\sigma_{1}(W)} \sum_{i, j} W^{i j} S\left(g_{0}\right)_{i j}+\frac{\varepsilon \sigma_{1}\left(g_{0}\right)}{\sigma_{1}^{2}(W)}
\end{aligned}
$$

Going back to (6.25), we have

$$
\begin{aligned}
- & 2 \alpha e^{-2 u} \frac{\sigma_{2}(W)-\varepsilon}{\sigma_{1}(W)}+O(1) \\
& \geq F_{t}-\sum_{i, j} A^{i j} F_{i j}-2 \alpha \mathrm{e}^{-2 u} u_{t} \\
& \geq\left(\alpha-r_{\varepsilon}(g)\right) \mathrm{e}^{-2 u}\left[\frac{-2 \sigma_{2}(W)-2 \varepsilon}{\sigma_{1}(W)}+\frac{2\left(\sigma_{1}^{2}(W)-\sigma_{2}(W)\right) \sigma_{1}\left(g_{0}\right)}{\sigma_{1}^{2}(W)}\right. \\
& \left.-\frac{2}{\sigma_{1}(W)} \sum_{i, j} W^{i j} S\left(g_{0}\right)_{i j}+\frac{2 \varepsilon \sigma_{1}\left(g_{0}\right)}{\sigma_{1}^{2}(W)}\right]
\end{aligned}
$$

since $\left(A^{i j}\right)$ is positive definite and $\alpha-r_{\varepsilon}(g)>Y_{2,1}\left(\left[g_{0}\right]\right)$ is positive. One can check $\sigma_{2}(g)=O(1)$ for $\|u\|_{C^{2}}$ is uniformly bounded and $\sum_{i, j}, W^{i j} S\left(g_{0}\right)_{i j}=$ $O(1)$. Also the term $\sigma_{1}^{2}(W)-\sigma_{2}(W)$ is always non-negative. From (6.27), we conclude that there is a positive constant $C_{2}>0$ (independent of $T$ ) such that

$$
\sigma_{1}(W)\left(x_{0}, t_{0}\right)>C_{2}>0
$$


Now for any $(x, t) \in M \times[0, T]$, we have

$$
\frac{\sigma_{2}(W)(x, t)-\varepsilon}{\sigma_{1}(W)(x, t)} \geq O(1),
$$

so that there is a positive constant $C>0$, independent of $T$, such that

$$
\sigma_{1}(W)(x, t) \geq C
$$

since we have $\sigma_{2}(W) \leq \frac{1}{2} \sigma_{1}^{2}(W)$ provided $\sigma_{2}(W) \geq 0$. This finishes the proof of the proposition.

Step 3. Now we can prove equation (6.10) admits a solution. From Steps 1 and 2, we know that the flow is uniformly parabolic. Krylov's theory implies $u(t)$ has a uniform $C^{2, \alpha}$ bound. Hence, $T^{*}=\infty$. One can also show that $u(t)$ globally converges to $u(\infty)$, which clearly is a solution of $(6.10)$ for $k=r_{\varepsilon}(g(\infty))$. (Note that $r_{\varepsilon}(g(t))$ is monotone and bounded, so that $r_{\varepsilon}(g(\infty))$ exists) (see [20]). So $u_{\varepsilon}=u(\infty)-\frac{1}{2} \log \left|r_{\varepsilon}(g(\infty))\right|$ solves (6.10) for $\kappa=1$ (resp. 0,1) if $r_{\varepsilon}(g(\infty))>0$ (resp. $=0,<0$ ). Now, if $\varepsilon$ is sufficiently small, we have $r_{\varepsilon}(g(0))>0$. Thus, there exists a minimizing solution to (6.10), that is, $a_{\varepsilon}$ is achieved.

Proof of Theorem 1 in the case $n=3$. Now let $u_{\varepsilon}$ be a minimizing solution to (6.10) for $\kappa=1$. It is clear $e^{-2 u_{\varepsilon}} g_{0} \in \mathcal{C}_{2}\left(\left[g_{0}\right]\right)$ and $\tilde{Y}_{2,1}\left(\left[g_{0}\right]\right) \geq a_{\varepsilon}$. As $a_{\varepsilon} \rightarrow$ $Y_{2,1}\left(\left[g_{0}\right]\right)$, we infer $\tilde{Y}_{2,1}\left(\left[g_{0}\right]\right) \geq Y_{2,1}\left(\left[g_{0}\right]\right)$. On the other hand, $\tilde{Y}_{2,1}\left(\left[g_{0}\right]\right) \leq$ $Y_{2,1}\left(\left[g_{0}\right]\right)$ since $\mathcal{C}_{2}\left(\left[g_{0}\right]\right) \subset \mathcal{C}_{1}\left(\left[g_{0}\right]\right)$. Finally, $\tilde{Y}_{2,1}\left(\left[g_{0}\right]\right)=Y_{2,1}\left(\left[g_{0}\right]\right)$. This finishes the proof of theorem.

\section{Acknowledgments}

The second named author is partly supported by SFB/TR71 of DFG.

\section{References}

[1] T. Aubin, Équations différentilles non linéaires et probléme de Yamabe concernant la courbure scalaire, J. Math. Pures Appl. 55 (1976), 269-296.

[2] K. Akutagawa and A. Neves, 3-manifolds with Yamabe invariant greater than that of $\mathbb{R P}^{3}$, J. Differ. Geom. 75(3) (2007), 359-386.

[3] Bray, Hubert L.; Neves, André, Classification of prime 3-manifolds with Yamabe invariant greater than $\mathbb{R P}^{3}$, Ann. Math. (2) 159 (2004), 407-424. 
[4] S. Brendle and J. Viaclovsky, A variational characterization for $\sigma_{n / 2}$, Calc. Var. P. D. E. 20 (2004), 399-402.

[5] A. Chang, M. Gursky and P. Yang, An equation of Monge-ampère type in conformal geometry, and four manifolds of positive Ricci curvature, Ann. Math. 155 (2002), 709-787.

[6] A. Chang, M. Gursky and P. Yang, An a priori estimates for a fully nonlinear equation on Four-manifolds, J. D'Analysis Math. 87 (2002), $151-186$.

[7] A. Chang, M. Gursky and P. Yang, Entire solutions of a fully nonlinear equation, Lectures on partial differential equations, 43-60, New Stud. Adv. Math. 2, Int. Press, Somerville, MA, 2003.

[8] S. Chen, Local estimates for some fully nonlinear elliptic equations, Int. Math. Res. Not. 2005 (2005), 3403-3425.

[9] B. Chow, P. Lu and L. Ni, Hamilton's Ricci flow, Graduate Studies in Mathematics, 77. American Mathematical Society, Providence, RI; Science Press, New York (2006).

[10] C. De Lellis and P. Topping, Almost Schur theorem, Calc. Var. PDE 43(3-4) (2012), 347-354

[11] Y. Ge, C.-S. Lin and G. Wang, On $\sigma_{2}$-scalar curvature, J. Diff. Geom. 84 (2010), 45-86.

[12] Y. Ge and G. Wang, On a fully nonlinear Yamabe problem, Ann. Sci. École Norm. Sup. 39 (2006), 569-598.

[13] Y. Ge and G. Wang, On a conformal quotient equation, Int. Math. Res. Not. IMRN 2007, Art, ID rnm019, 32pp.

[14] Y. Ge and G. Wang, An almost Schur theorem on 4-dimensional manifolds, PAMS. 140 (2012) 1041-1044.

[15] Y. Ge and G. Wang, A new conformal invariant on 3-dimensional manifolds, preprint 2011.

[16] Y. Ge, G. Wang and C. Xia, On problems related to an inequality of Andrews, De Lellis and Topping, Int Math Res Not. IMRN, to appear, doi:10.1093/imrn/rns196

[17] M.d.M. González, Removability of singularities for a class of fully nonlinear elliptic equations, Calc. Var. PDE, 27 (2006), 439-466. 
[18] P. Guan, C.-S. Lin and G. Wang, Local gradient estimates for conformal quotient equations, Int J. Math. 18 (2007), 349-361.

[19] P. Guan and G. Wang, Local estimates for a class of fully nonlinear equations arising from conformal geometry, Int. Math. Res. Not. 2003, (2003), 1413-1432.

[20] P. Guan and G. Wang, A fully nonlinear conformal flow on locally conformally flat manifolds, J. R. Angew. Math. 557 (2003), 219-238.

[21] P. Guan and G. Wang, Geometric inequalities on locally conformally flat manifolds, Duke Math. J. 124 (2004), 177-212.

[22] M. Gursky and J. Viaclovsky, Volume comparison and the $\sigma_{k}$-Yamabe problem, Adv. Math. 187 (2004), 447-487.

[23] M. Gursky and J. Viaclovsky, A fully nonlinear equation on 4-manifolds with positive scalar curvature, J. Diff. Geom. 63 (2003), 131-154.

[24] M. Gursky and J. Viaclovsky, Prescribing symmetric functions of the eigenvalues of the Ricci tensor, Ann. Math., 166 (2007), 475-531.

[25] E. Hebey, Nonlinear analysis on manifolds: Sobolev spaces and inequalities. Courant Lecture Notes in Math., 5. Courant Inst. of Math. Sci., New York; Amer. Math. So., Providence, RI, 1999.

[26] O. Kobayashi, Scalar curvature of a metric with unit volume, Math. Ann. 279 (1987), 253-265.

[27] A. Li and Y. Li, On some conformally invariant fully nonlinear equations, Comm. Pure Appl. Math., 56 (2003), 1416-1464.

[28] A. Li and Y. Li, A Liouville type theorem for some conformally invariant fully nonlinear equations, Geometric analysis of PDE and several complex variables, 321-328, Contemp. Math., 368, Amer. Math. Soc., Providence, RI, 2005.

[29] R. Schoen, Conformal deformation of a Riemannian metric to constant curvature, J. Diff. Geome. 20 (1984), 479-495.

[30] R. Schoen, Variational theory for the total scalar curvature functional for Riemannian metrics and related topics, Lecture Notes in Math. 1365, 120-154, Springer-Verlag, New York, 1989.

[31] L. Simon, Asymptotics for a class of nonlinear evolution equations, with applications to geometric problems, Ann. Math. 118 (1983), 525-571. 
[32] N. Trudinger, On imbeddings into Orlicz spaces and some applications, J. Math. Mech. 17 (1967), 473-483.

[33] J. Viaclovsky, Conformal geometry, contact geometry and the calculus of variations, Duke J. Math. 101 (2000), no. 2, 283-316.

[34] J. Viaclovsky, Conformally invariant Monge-Ampère equations: global solutions, Trans. AMS 352 (2000), 4371-4379.

[35] J. Viaclovsky, Estimates and some existence results for some fully nonlinear elliptic equations on Riemannian manifolds, Commun. Anal. Geom. 10 (2002), 815-847.

[36] H. Yamabe, On a deformation of Riemannian structures on compact manifolds, Osaka Math. J., 12 (1960), 21-37.

Laboratoire D'Analyse et de Mathématiques Appliquées

CNRS UMR 8050

DÉPARTEMENT DE MATHÉMATiques

Université Paris XII-VAL DE MARne

61 avenue du Général de Gaulle

94010 Créteil Cedex,

FRANCE

E-mail address: ge@u-pec.fr

Albert-Ludwigs-Universität Freiburg

Mathematisches Institut

ECKERSTR. 1, D-79104 FreiburG

Germany

E-mail address: guofang.wang@math.uni-freiburg.de

RECEIVED MARCH 13, 2012 